\title{
Asset Pricing with Uncertainty About the Long Run*
}

\author{
Hui Chen ${ }^{\dagger}$ \\ Massachusetts Institute of Technology \\ Michal Pakoš \\ Carnegie Mellon University
}

June 13, 2008

\begin{abstract}
When investors have preferences for early resolution of uncertainty, higher uncertainty about future growth rates raises agents' marginal utility, which generates a premium for bearing uncertainty risk. If expected growth rates have persistent long-run dynamics, small local uncertainty about the growth rate can translate into large uncertainty about future cash flows, which amplify the uncertainty premium. We study the effects of learning on the dynamics of asset prices in an endowment economy, where agents learn about consumption growth through a nonlinear filter. The uncertainty premium is large, varies substantially over time, and tends to rise following negative consumption shocks. Moreover, a decrease in consumption volatility can sometimes sharply increase the Sharpe Ratio and risk premium.
\end{abstract}

Keywords: Long-Run Risks; Predictability; Uncertainty Premium; Wonham Filter

${ }^{*}$ We thank Ravi Bansal, Lars Hansen, Monika Piazzesi, Steve Shreve, seminar participants at Bank of Canada and Boston University for helpful discussions. This draft is very preliminary. Please do not circulate without the consent of the authors.

${ }^{\dagger}$ MIT Sloan School of Management, 50 Memorial Drive E52-447, Cambridge, MA 02142; ph: (617) 324-3896; email: huichen@mit.edu

${ }^{\ddagger}$ Tepper School of Business, Carnegie Mellon University, 5000 Forbes Avenue, Pittsburgh PA 15213; ph: (412) 268-4063; email: misko@andrew.cmu.edu 


\section{Introduction}

This paper demonstrates a rational channel that connects uncertainty, volatility, and returns. When investors have preferences for early resolution of uncertainty, changes in the uncertainty about future growth prospects of the economy affect agents' marginal utility. These investors dislike assets that pay off poorly at times of high uncertainty, and demand an uncertainty premium for holding such assets. If expected growth rates have persistent long-run dynamics, small local uncertainty about the growth rate can translate into large uncertainty about cash flows in the long run, which dramatically amplify the uncertainty premium as well as price volatility.

We study these effects quantitatively in an endowment economy with imperfect information. The model setup is similar to Veronesi (2000): the expected growth rate of consumption follows a Markov-switching process, and agents learn about the growth rate through the nonlinear filter of Wonham (1964). An attractive feature of the Wonham filter is that it generates stochastic uncertainty, unlike the deterministic decay of uncertainty for the Kalman-Bucy filter. Agents in our model have recursive preferences, which allows for preferences for early resolution of uncertainty, and we introduce highly persistent dynamics in consumption growth rates as motivated by the long-run risk literature. The model generates a large uncertainty premium, which varies over time, and tends to rise significantly following negative consumption shocks. Moreover, a decrease in consumption volatility can sometimes sharply raise the Sharpe Ratio and risk premium.

Our model provides a micro foundation for the variance premium studied by Bollerslev and Zhou (2007), and Drechsler and Yaron (2007). Bollerslev and Zhou show that the variance premium, defined as the difference between the implied and realized variances, has large, statistically significant predictive power for stock returns. In this model, high variance in returns occurs when uncertainty is high. Thus, an asset that pays off a lot in those states with high realized variance of returns is a hedge for the uncertainty risk, which explains why the variance premium is almost always positive. Moreover, since the variance premium rises with uncertainty, it will predict returns.

Why is learning important in the presence of long-run risks? Intuition might suggest the opposite, since high persistence of the predictable component in consumption growth makes inference about consumption growth rates very difficult. However, posterior belief about the growth rate will also inherit the persistence in the expected growth rate. Thus, small revisions in the agent' belief of the state lead to dramatic changes in her projected long-run consumption paths. This means that a small amount of uncertainty about current growth rate will be magnified into large uncertainty about future consumption, and agents will demand more compensation for bearing such risks.

Our simple model is also able to generate return predictability in the right direction. The uncertainty premium is hump-shaped and tends to peak when the posterior probability of the good state is near 0.5 , which suggest that a negative consumption shock can lead to either higher or lower risk premium, depending on which side of the bell curve we are on. However, since the economy spends more time in the good state, the Markov Chain is asymmetric. This can leads to a highly skewed steady-state distribution of the posterior. As a result, most of the time we are in a region 
where uncertainty premium rises following negative consumption shocks.

Another interesting finding of the paper is that lower consumption volatility can lead to higher Sharpe ratio and risk premium for stocks. When volatility of consumption becomes smaller, the signals about the hidden growth rate becomes more precise. As a result, agents will respond to consumption shocks more aggressively, leading to larger volatility in the posterior probability $\pi$, which in turn raises the risk price for uncertainty, as well as the volatility of returns. The two effects combine to lead to higher risk premium. This effect is consistent with the finding of Veronesi (2000), although in his model higher information quality leads to higher risk premium entirely through volatility, and has no effect on risk prices (Sharpe ratio).

Finally, we also study the effects of uncertainty risk on the term structure of real interest rates. Our model explains such stylized facts as the level and the time series properties of the real interest rates and the long term bond premium.

There is a large literature on learning in financial markets. Earlier work in this area include Gennotte (1986), Detemple (1986), Dothan and Feldman (1986), Wang (1993), David (1997), Veronesi (1999), Veronesi (2000), Brennan and Xia (2001), etc. More recent work include Ai (2007).

Veronesi (2000) also studies the effects of learning on asset pricing in an endowment economy. He considers CRRA preferences 1 In this case, the Sharpe ratio is constant (proportional to the coefficient of relative risk aversion and volatility of consumption), and risk premium moves in lock steps with the systematic volatility in returns. Veronesi's model highlights the tension between the coefficient of relative risk aversion (RRA) and the intertemporal elasticity of substitution (IES). On the one hand, we need high RRA to raise the Sharpe ratio. On the other hand, high RRA makes IES less than 1. In the presence of learning, this induces a negative covariation between consumption shocks and returns, which drives risk premium lower and often times negative! In fact, Veronesi (2000) (page 824) conjectures that "the exact balance between the two effects can only be assessed by using a more general utility function, such as Epstein and Zin (1989)." In this paper, we not only introduces recursive preferences, but also emphasize the effects of long-run risk.

Hansen (2007) suggest that risk prices for uncertainty can be important; he calibrates the consumption growth rate with business cycle dynamics, which also serves as the benchmark calibration in this paper. The business cycle dynamics of consumption growth are not sufficiently persistent, and generate small risk prices and uncertainty premium. We show that this effect gets amplified when the dynamics of consumption transcend the business cycle. We also show that uncertainty premium contributes to return predictability and has interesting effects on the term structure.

Our paper is also related to the long-run risk literature. Bansal and Yaron (2004) show that with recursive preferences, a small long-run predictable component in consumption growth can generate a large equity premium. Agents not only care about local consumption risks, but also the fluctuations of consumption in the future. To make risk premium vary over time, they introduce

\footnotetext{
${ }^{1}$ Hung (1994) also studies a discrete-time Lucas-tree economy where the fundamentals - consumption and dividends - follow a bivariate regime switching model, and investors are endowed with recursive Epstein-Zin preferences. He assumes that investors observe the true state of the economy.
} 
heteroskedasticity in consumption growth. A large body of subsequent studies shows that long-run risk models can explain a wide range of asset pricing phenomena: Bansal, Dittmar, and Lundblad (2005), Kiku (2006) on the cross section of stock returns; Colacito and Croce (2006) on exchange rates; Piazzesi and Schneider (2005) on long-term nominal bond risk premia; Chen (2007) on credit spreads, etc.

A main challenge of this line of research is to find empirical evidence of long-run consumption dynamics. Hansen, Heaton, and Li (2005), Bansal, Kiku, and Yaron (2006), and Colacito and Croce (2006) provide empirical evidence of long-run component in consumption growth. However, evidence on stochastic volatility in consumption growth is weaker. We show that even if consumption growth is homoskedastic, time-varying uncertainty through filtering alone can generate large variation in the risk premium.

Duffie, Schroder, and Skiadas (1997) study the impact of timing of resolution of uncertainty on the term structure of interest rates. Their filtering problem generates deterministic decay in uncertainty, and they do not study the impact of long run risks. Piazzesi and Schneider (2006) introduce long run risks to the term structure models. Their model features adaptive learning.

There are two recent papers study the effect of learning in long-run risk models. Ai (2007) analyzes the welfare implications of learning in a model with long run risks. Bansal and Shaliastovich (2008) show that learning endogenously generate asset price jumps in a long-run risks based equilibrium.

\subsection{Timing of Resolution of Uncertainty}

With time-separable utility, marginal utility is only affected by current period consumption; with recursive preferences, the entire trajectory of future consumption matters. Risks can no longer be captured by one-period consumption volatility. To illustrate the idea of the timing of uncertainty resolution with a simple example from the work of Chew and Epstein (1989). Consider a two-period economy with two risky assets, whose payoffs are shown in Figure 1 .

The payoffs for both assets at $t=1$ are determined by a coin-flip, and the two possible outcomes are $c_{1}(1)>c_{1}(2)$. For the first asset, the coin is flipped at time $t=0$, whereas for the second asset, the coin is flipped at $t=1$.

Suppose the coin is biased, with the probabilities of head and tail $\alpha$ and $1-\alpha$. The utility from buying the first asset is 2

$$
U\left(\alpha \delta\left[c_{0}, c_{1}(1)\right]+(1-\alpha) \delta\left[c_{0}, c_{1}(1)\right]\right)
$$

Let us define the probability $\beta$ in such a way that the investor is indifferent between buying either asset, that is,

$$
U\left(\alpha \delta\left[c_{0}, c_{1}(1)\right]+(1-\alpha) \delta\left[c_{0}, c_{1}(2)\right]\right):=: U\left(\delta\left[c_{0}, \beta c_{1}(1)+(1-\beta) c_{1}(2)\right]\right)
$$

\footnotetext{
${ }^{2}$ The notation purposefully follows Chew and Epstein (1989)
} 


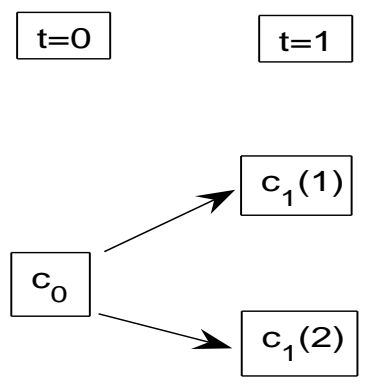

Early Resolution of Uncertainty
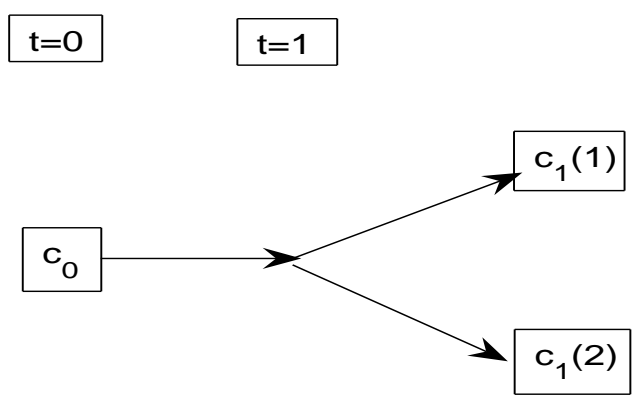

Late Resolution of Uncertainty

Figure 1: Example: Timing of Resolution of Uncertainty

The interpretation is that in equilibrium the investor must hold both assets. Chew and Epstein (1989, p. 109) prove that if the investor prefers early to late resolution of uncertainty, the probability $\beta$ must be larger than $\alpha$. In other words, the expected return on the second asset must be larger than the expected return on the first one

$$
\mathbf{E}\left[R_{1}\right] \equiv \frac{\alpha c_{1}(1)+(1-\alpha) c_{1}(2)}{c_{0}}<\frac{\beta c_{1}(1)+(1-\beta) c_{1}(2)}{c_{0}} \equiv \mathbf{E}\left[R_{2}\right]
$$

This simple example suggests that risk premia on assets are crucially affected by the way the uncertainty about their payoffs unfolds. In fact, as we show hereafter, risk premia in expected returns on fixed income and equities contain an "uncertainty premium (discount)" when agents prefer early (late) resolution of uncertainty. The asset pricing effects of preferences for the timing of resolution of uncertainty are missed by expected utility specifications. In this paper, we demonstrate quantitatively their important effects on asset pricing in the existence of long-run risks.

\section{The Economy}

Consider an endowment economy (Lucas (1978)) with a single perishable consumption good and a continuum of identical investors. 
Preferences Investors are endowed with stochastic differential utility of Duffie and Epstein (1992), which is a continuous-time version of the recursive preferences of Kreps and Porteus (1978), Epstein and Zin (1989) and Weil (1990). The utility index at time $t$ over consumption process $c$ is:

$$
U_{t}=\mathbb{E}_{t}\left(\int_{t}^{\infty} f\left(c_{s}, U_{s}\right) d s\right)
$$

where $f(c, v)$ is a normalized aggregator of consumption and continuation value. It is defined as:

$$
f(c, v)=\frac{\delta}{1-\frac{1}{\psi}} \frac{c^{1-\frac{1}{\psi}}-((1-\gamma) v)^{\frac{1-1 / \psi}{1-\gamma}}}{((1-\gamma) v)^{\frac{1-1 / \psi}{1-\gamma}-1}}
$$

where $\delta$ is the rate of time preference, $\gamma$ determines the coefficient of relative risk aversion for timeless gambles, and $\psi$ determines the elasticity of intertemporal substitution for deterministic consumption paths. To simplify notation, we define $\rho=1-\frac{1}{\psi}$ and $\alpha=1-\gamma$.

For stochastic differential utility, Skiadas (1998) shows that when the temporal aggregator $f$ is convex (concave), the utility index $U$ will be increasing (decreasing) in information. This condition implies that agents prefer early (late) resolution of uncertainty when $\gamma>(<) \frac{1}{\psi}$. In the special case when $\gamma=\frac{1}{\psi}$, the aggregator becomes additive, and an agent is indifferent towards the timing of resolution. These results are consistent with the conditions for discrete time recursive utility (Epstein and Zin 1989, p. 952).

Endowment Aggregate consumption $C$ evolves as:

$$
\frac{d C}{C}=\theta d t+\sigma_{c} d Z^{c}
$$

where $\theta$ is the expected growth rate, $\sigma_{c}$ is the constant local consumption volatility, $Z^{c}$ is a standard Brownian motion.

The expected growth rate $\theta$ follows a continuous-time hidden Markov chain with two states $\bar{\theta}>\underline{\theta}$; its transition matrix for the time interval $(t, t+d t)$ is:

$$
\mathbb{P}(d t)=\left(\begin{array}{cc}
1-\lambda d t & \lambda d t \\
\mu d t & 1-\mu d t
\end{array}\right) .
$$

The transition densities $\lambda$ and $\mu$ determine how persistent each state of the Markov chain is.

Aggregate consumption consists of two parts. The first part is the dividend $D$ of a risky asset (levered stock),

$$
\frac{d D}{D}=\left[\theta_{m}+\phi\left(\theta-\theta_{m}\right)\right] d t+\phi \sigma_{c} d Z^{c}+\sigma_{d} d Z^{d}
$$


where $\theta_{m}$ is the long-run average growth rate of consumption,

$$
\begin{aligned}
\theta_{m} & =\pi^{s} \bar{\theta}+\left(1-\pi^{s}\right) \underline{\theta} \\
\pi^{s} & =\frac{\mu}{\lambda+\mu}
\end{aligned}
$$

and $Z^{d}$ is an independent standard Brownian motion, which provides additional independent shocks to the dividends. The difference between consumption and dividend is other sources of income, which we do not model explicitly in this paper.

This specification of dividend is motivated by the "levered stock" of Abel (1999). We restrict dividend and consumption to have the same long-run growth rate, but dividend is more exposed to the long-run predictable component $\theta$, as well as consumption shocks. The parameter $\phi$ controls the leverage effect.

Signals Past realizations of consumption and dividend are public signals that help investors learn about the hidden consumption growth rate. Investors could be observing other useful signals as well. To introduce additional source of variation in information quality, we follow Veronesi (2000) and assume that investors have an additional noisy signal of the growth rate:

$$
d e=\theta d t+\sigma_{e} d Z^{e}
$$

where $Z^{e}$ is a standard Brownian motion independent of $Z^{c}$ and $Z^{d}$.

\section{$2.1 \quad$ Learning}

Let $\mathcal{I}(t)$ be investors' information set at time $t$, which includes the past history of $\left\{\frac{d C}{C}, \frac{d D}{D}\right.$, de $\}$ up to time $t$. Also, let $\pi(t)$ be investors' belief that the expected growth rate at time $t$ is equal to $\bar{\theta}$ :

$$
\pi(t)=\mathbb{P}\{\theta(t)=\bar{\theta} \mid \mathcal{I}(t)\}
$$

For investors, the mean of the expected growth rate at time $t$ is:

$$
\widehat{\theta}(t)=\pi(t) \bar{\theta}+(1-\pi(t)) \underline{\theta} .
$$

Investors update their belief $\pi$ based on the signals $\left\{\frac{d C}{C}, \frac{d D}{D}, d e\right\}$. Its dynamics is summarized in the following lemma.

Lemma 1 The posterior probability $\{\pi(t), \mathcal{I}(t)\}_{t \geq 0}$ follows a time-homogenous diffusion

$$
d \pi=(\lambda+\mu)\left(\pi^{s}-\pi\right) d t+v(\pi)\left(\frac{1}{\sigma_{c}} d \widetilde{Z}^{c}+\frac{1}{\sigma_{e}} d \widetilde{Z}^{e}\right)
$$

where $\pi^{s}=\mu /(\lambda+\mu)$ is the long-run probability of good state (implied by the stationary distribu- 
tion),

$$
v(\pi)=(\bar{\theta}-\underline{\theta}) \pi(1-\pi),
$$

and

$$
\begin{aligned}
d \widetilde{Z}^{c} & =\frac{1}{\sigma_{c}}\left(\frac{d C}{C}-\widehat{\theta} d t\right) \\
d \widetilde{Z}^{e} & =\frac{1}{\sigma_{e}}(d e-\widehat{\theta} d t)
\end{aligned}
$$

are independent standard Brownian motions with respect to the filteration $\mathcal{I}(t)$.

Proof. Liptser and Shiryayev (2001), Theorem 9.1.

The dynamics of $\pi$ intuitively describes the learning problem. The drift coefficient of the process suggests that the posterior belief is expected to converge to the stationary distribution. The speed of mean reversion depends on the persistence of the Markov chain: the more persistent the Markov chain, the slower this convergence. In addition, investors will adjust their beliefs in the direction of the "surprises" from the signals. They will adjust their belief more in response to the surprises when the precisions of the signals $\left(1 / \sigma_{c}, 1 / \sigma_{e}\right)$ are high, and when their prior uncertainty about the state $(v(\pi))$ is high. In Appendix $\AA$, we characterize the steady-state distribution of the posterior probability using the Kolmogorov forward equation.

It may come as a surprise that realized dividend growth does not appear in the filtering problem. Dividend growth is also a noisy signal of the hidden growth rate of consumption, and should provide information about the hidden state. However, we assume that the time-varying component of expected dividend growth and the systematic volatility are their counterparts for consumption raised by a common factor $\phi$. This assumption makes dividend growth a redundant signal. Intuitively, we can rewrite $d D / D$ as:

$$
\frac{d D}{D}=(1-\phi) \theta_{m} d t+\phi \frac{d C}{C}+\sigma_{d} d Z^{d} .
$$

Thus, realized dividend growth is a linear function of the realized consumption growth plus independent noises, and it does not contain additional information about $\theta$ that is not already included in realized consumption growth 3

Under investors' information set, the consumption and dividend dynamics can be rewritten as:

$$
\frac{d C}{C}=\widehat{\theta}(t) d t+\sigma_{c} d \widetilde{Z}^{c}
$$

and

$$
\frac{d D}{D}=\left[\theta_{m}+\phi\left(\widehat{\theta}(t)-\theta_{m}\right)\right] d t+\phi \sigma_{c} d \widetilde{Z}^{c}+\sigma_{d} d Z^{d} .
$$

\footnotetext{
${ }^{3}$ We also solved the model with a more general dividend process, allowing the systematic volatility of dividend growth to be consumption volatility multiplied by a factor different from $\phi$. The results are qualitatively similar.
} 


\subsection{Local versus Long-run Uncertainty}

With recursive preferences, investors care about both current and future consumption. Thus, it is important to understand uncertainty about current growth rate, which we refer to as local uncertainty, and uncertainty about the long-run growth rates, which we refer to as long-run uncertainty. We define local uncertainty as:

$$
V(t, t)=\operatorname{Var}[\theta(t) \mid \mathcal{I}(t)]=(\bar{\theta}-\underline{\theta})^{2} \pi(t)(1-\pi(t)),
$$

which measures investors' uncertainty about the instantaneous growth rate. The local uncertainty depends on how far apart the two states are, and it peaks when investors assign equal probability to the good and bad state.

We define long-run uncertainty as:

$$
V(t, T)=\operatorname{Var}\left[\int_{t}^{T} \theta(s) d s \mid \mathcal{I}(t)\right],
$$

which measures investors' uncertainty about the accumulative growth rate over a given period of time. The long-run uncertainty can be computed in closed form, as shown below.

Lemma 2 The long-run uncertainty is equal to

$$
\begin{aligned}
V(t, T)= & \underline{\theta} \iota_{2}^{\prime} \mathbf{U} \mathbf{A}(t, T) \mathbf{U}^{-1}[\bar{\theta}, \underline{\theta}]^{\prime}+[\bar{\theta},-\underline{\theta}] \mathbf{A}(t, T) \mathbf{U}^{-1}[\bar{\theta}, \underline{\theta}]^{\prime} \pi^{s} \\
& -[\bar{\theta},-\underline{\theta}] \mathbf{U B}(t, T) \mathbf{U}^{-1}[\bar{\theta}, \underline{\theta}]^{\prime}\left(1-\pi_{t}\right) \\
& -\left[\underline{\theta}(T-t)+(\bar{\theta}-\underline{\theta})(T-t) \pi^{s}+\left(\frac{\bar{\theta}-\underline{\theta}}{\lambda+\mu}\right)\left(\pi_{t}-\pi^{s}\right)\left(1-e^{-(\lambda+\mu)(T-t)}\right)\right]^{2},
\end{aligned}
$$

where $\mathbf{U}, \mathbf{A}(t, T)$ and $\mathbf{B}(t, T)$ are given in Appendix.

Proof. See Appendix.

\section{Pricing Kernel and Asset Prices}

Let the value function of the representative agent be $J$. At each $t$, she consumes $c$, invests a fraction $x$ of wealth in the aggregate consumption claim, which has dividend payment equal to aggregate consumption, and the rest in a riskless bond (in zero net supply) with interest rate $r$. Let $P$ be the price of the consumption claim. Then, the representative agent's problem is:

$$
J(t)=\max _{c, x} \mathbb{E}\left\{\int_{t}^{\infty} f(c(\tau), J(\tau)) d \tau \mid \mathcal{I}(t)\right\},
$$


subject to the dynamic budget constraint:

$$
d W=W\left[x\left(\frac{d P+C d t}{P}\right)+(1-x) r d t\right]-c d t .
$$

In equilibrium, the representative agent must be holding only the aggregate consumption claim and none of the riskless bond, which imply the market-clearing conditions: $x(t)=1, c(t)=C(t)$. This result also implies that the value of the consumption claim is equal to the agent's wealth: $P(t)=W(t)$. The following two propositions summarize the equilibrium results.

Proposition 1 The value function $J$ takes the form:

$$
\left.J(W, \pi)=\alpha^{-1} \delta^{(\alpha / \rho)}[\Psi(\pi)]\right]^{\left(\frac{1-\rho}{\rho / \alpha}\right)} W^{\alpha},
$$

where the function $\Psi:(0,1) \rightarrow \mathbb{R}^{+}$equals the equilibrium wealth-consumption ratio:

$$
\Psi(\pi)=\frac{W}{C}
$$

and solves the following boundary value problem:

$$
0=1+a_{0}(\pi) \Psi+a_{1}(\pi) \Psi^{\prime}+a_{2}(\pi) \frac{\left(\Psi^{\prime}\right)^{2}}{\Psi}+a_{3}(\pi) \Psi^{\prime \prime},
$$

subject to two boundary conditions at $\partial(0,1)$ :

$$
\begin{aligned}
& 0=1+\left[-\delta+\rho \underline{\theta}-\frac{1}{2} \rho(1-\alpha) \sigma_{c}^{2}\right] \Psi(0)+\mu \Psi^{\prime}(0) \\
& 0=1+\left[-\delta+\rho \bar{\theta}-\frac{1}{2} \rho(1-\alpha) \sigma^{2}\right] \Psi(1)-\lambda \Psi^{\prime}(1)
\end{aligned}
$$

The functions $a_{0}(\pi), a_{1}(\pi), a_{2}(\pi)$ and $a_{3}(\pi)$ are given in Appendix.

Proof. See Appendix.

Given the wealth-consumption ratio $\Psi(\pi)$, it is straightforward to solve for the pricing kernel:

Proposition 2 Under the information set of the representative agent, the pricing kernel, $m$, satisfies:

$$
\frac{d m_{t}}{m_{t}}=-r(\pi) d t-\eta_{c}(\pi) d \widetilde{Z}^{c}-\eta_{e}(\pi) d \widetilde{Z}^{e},
$$

where $r(\pi)$ is the riskfree real rate:

$$
\begin{aligned}
r(\pi)= & \delta+\frac{1}{\psi} \mathbb{E}_{t}\left(\frac{d C}{C}\right)-\frac{1}{2} \gamma\left(1+\frac{1}{\psi}\right) \operatorname{Var}_{t}\left(\frac{d C}{C}\right) \\
& -\left(1-\frac{1-\gamma}{1-\frac{1}{\psi}}\right) \frac{\Psi^{\prime}}{\Psi} \operatorname{Cov}_{t}\left(\frac{d C}{C}, d \pi\right)-\frac{1}{2}\left(1-\frac{1-\gamma}{1-\frac{1}{\psi}}\right)\left(\frac{\Psi^{\prime}}{\Psi}\right)^{2} \operatorname{Var}_{t}(d \pi) .
\end{aligned}
$$


The risk price for filtered consumption shocks is:

$$
\eta_{c}(\pi)=\gamma \sigma_{c}+\left(1-\frac{1-\gamma}{1-\frac{1}{\psi}}\right) \frac{\Psi^{\prime}}{\Psi}\left(\frac{\bar{\theta}-\underline{\theta}}{\sigma_{c}}\right) \pi(1-\pi),
$$

and the risk price for filtered signal shocks is:

$$
\eta_{e}(\pi)=\left(1-\frac{1-\gamma}{1-\frac{1}{\psi}}\right) \frac{\Psi^{\prime}}{\Psi}\left(\frac{\bar{\theta}-\underline{\theta}}{\sigma_{e}}\right) \pi(1-\pi) .
$$

Proof. See Appendix.

The first three terms for the riskfree rate are standard. When consumption growth is iid, the riskfree rate is constant,

$$
r=\delta+\frac{1}{\psi} \theta-\frac{1}{2} \gamma\left(1+\frac{1}{\psi}\right) \sigma_{c}^{2}
$$

A high subjective rate of time preference $\delta$ and a high expected consumption growth rate $\mathbb{E}_{t}(d C / C)$ make investors want to borrow against their future endowment stream, which leads to a rise in the spot interest rate, to the point where everyone is content to consume their endowment stream. This effect depends inversely on investors' willingness substitute consumption over time, controlled by the elasticity of intertemporal substitution $\psi$.

The remaining three terms of $r$ represent the effects of precautionary saving. Investors want to save more at times when consumption growth is volatile. If they prefer early resolution of uncertainty $(\gamma>1 / \psi)$, they will also want to save more when uncertainty about the growth rate is high, or beliefs about the growth rate are sensitive to consumption shocks. These demands for extra saving lead to a fall in the real interest rate. Thus, through precautionary savings motive, time-varying uncertainty can generate rich short rate dynamics.

Furthermore, the real interest rate overreacts to bad news in good times and underreacts to good news in bad times. Intuitively, suppose that $\pi$ is close to 1 and investors receive a bad piece of macroeconomic news. Such a piece of news is doubly bad. Not only does it mean a lower expected consumption growth rate but it also raises the uncertainty in the economy, and thereby strengthens the demand for precautionary savings (assuming investors prefer early resolution of uncertainty). Veronesi (1999) generates a similar feature for stocks in a general equilibrium model with constant short rate and exponential utility.

Next, the risk price for consumption shocks has two components. The first part is the standard risk price for local consumption shocks, which increases with risk aversion and the local volatility of consumption. The second part is the risk price for uncertainty about the growth rate. Recall that the local uncertainty about the growth rate is proportional to $\pi(1-\pi)$. Thus, risk prices tend to rise when uncertainty about the growth rate rises. In addition, the risk price for uncertainty depends on the equilibrium wealth-consumption ratio, which reflects the effects of long-run uncertainty. Shocks to the noisy signal are also priced, because they affect the posterior probability. The risk price for 
these shocks is tied to uncertainty as well.

One can show that $\Psi^{\prime}(\pi)>(<) 0$ when $\psi>(<) 1$. Then, it is easy to show that the risk price for uncertainty will be positive when $\gamma>\psi$, which is equivalent to the preferences for early resolution of uncertainty. With preferences for late resolution of uncertainty, the risk price for uncertainty will be negative. Finally, CRRA preferences are a special case of the recursive preferences where investors are indifferent to the timing of resolution of uncertainty. As shown in Veronesi (2000), in this case, there is no risk price for uncertainty, Sharpe ratio is constant, and uncertainty about the growth rate can only change the risk premium by changing return volatility.

The return process for the consumption claim satisfies:

$$
\frac{d P}{P}=\left(\mu_{P}(\pi)-\frac{C}{P}\right) d t+\sigma_{P, c}(\pi) d \widetilde{Z}^{c}+\sigma_{P, e}(\pi) d \widetilde{Z}^{e},
$$

where

$$
\begin{aligned}
\mu_{P}(\pi)-r(\pi) & =\eta_{c}(\pi) \sigma_{P, c}(\pi)+\eta_{e}(\pi) \sigma_{P, e}(\pi), \\
\sigma_{P, c}(\pi) & =\sigma_{c}+\frac{\Psi^{\prime}(\pi)}{\Psi(\pi)} \frac{v(\pi)}{\sigma_{c}}, \\
\sigma_{P, e}(\pi) & =\frac{\Psi^{\prime}(\pi)}{\Psi(\pi)} \frac{v(\pi)}{\sigma_{e}} .
\end{aligned}
$$

Similar to the decomposition of risk prices, the risk premium for consumption claims also has two components. The first part is compensation for exposure to consumption shocks, the second for exposure to signal shocks. Volatility of return not only depends on consumption volatility (cash-flow risk), but also an additional component related to uncertainty about the growth rate (uncertainty risk).

\section{1 "Levered" Stock}

The levered stock, with dividend following the process in equation (8), can be priced similarly. The results are summarized in the following proposition.

Proposition 3 The price of the "levered" stock is $S(D, \pi)$,

$$
S(D, \pi)=D \Pi(\pi)
$$

where the price dividend ratio $\Pi(\pi)$ solves the following boundary value problem:

$$
0=1+b_{0}(\pi) \Pi+b_{1}(\pi) \Pi^{\prime}+b_{2}(\pi) \Pi^{\prime \prime}
$$


The boundary conditions are:

$$
\begin{aligned}
& 0=1+\left[(1-\phi) \theta_{m}+\phi \underline{\theta}-r(0)-\eta_{c}(0) \phi \sigma_{c}\right] \Pi(0)+\mu \Pi^{\prime}(0) \\
& 0=1+\left[(1-\phi) \theta_{m}+\phi \bar{\theta}-r(1)-\eta_{c}(1) \phi \sigma_{c}\right] \Pi(1)-\lambda \Pi^{\prime}(1)
\end{aligned}
$$

where $r(\pi), \eta_{c}(\pi)$ and $\eta_{e}(\pi)$ are given in Proposition 2. The functions $b_{0}(\pi), b_{1}(\pi)$, and $b_{2}(\pi)$ are given in Appendix.

Proof. See Appendix.

The return process for the consumption claim satisfies:

$$
\frac{d S}{S}=\left(\mu_{S}(\pi)-\frac{D}{S}\right) d t+\sigma_{S, c}(\pi) d \widetilde{Z}^{c}+\sigma_{S, e}(\pi) d \widetilde{Z}^{e}+\sigma_{S, d} d Z^{d}
$$

where

$$
\begin{aligned}
\mu_{S}(\pi) & =r(\pi)+\eta_{c}(\pi) \sigma_{S, c}(\pi)+\eta_{e}(\pi) \sigma_{S, e}(\pi) \\
\sigma_{S, c}(\pi) & =\phi \sigma_{c}+\frac{\Pi^{\prime}(\pi)}{\Pi(\pi)} \frac{v(\pi)}{\sigma_{c}} \\
\sigma_{S, e}(\pi) & =\frac{\Pi^{\prime}(\pi)}{\Pi(\pi)} \frac{v(\pi)}{\sigma_{e}} \\
\sigma_{S, d} & =\sigma_{d} .
\end{aligned}
$$

\subsection{Real Zero-Coupon Bonds}

Kreps-Porteus recursive preferences play an important role also in understanding the uncertainty about the long-run consumption growth rate in the pricing of (indexed) real zero-coupon bonds. It is the relaxation of independence axiom of choice that leads to a preference for early resolution of uncertainty, and subsequently to interesting real bond dynamics, driven to a large extent by time-varying precautionary saving demand. The following proposition displays the fundamental asset pricing equation for the value of the real zero-coupon bonds of maturity $T$. Consistent with empirical evidence, to be reviewed hereafter, the model delivers bond return volatility clustering and time-varying conditional expected excess returns.

Proposition 4 Consider a real (indexed) zero-coupon bond with maturity $T$.

(i) The equilibrium price dynamics of the bond is given by the Ito process

$$
\frac{d B(t ; T)}{B(t ; T)}=\mu_{B}(t ; T) d t+\widetilde{\mathbf{\Sigma}}_{B}(t ; T) d \widetilde{\mathbf{Z}}(t)
$$

(ii) The equilibrium price of the bond $B(t ; T)=B(\pi(t), t ; T)$ solves the linear partial differential equation

$$
0=\partial_{t} B+\beta_{2}(\pi) \partial_{\pi \pi} B+\beta_{1}(\pi) \partial_{\pi} B+\beta_{0}(\pi) B
$$


Table 1: Model Calibration

\begin{tabular}{|c|c|c|c|c|}
\hline & $\bar{\theta}$ & $\underline{\theta}$ & $\lambda$ & $\mu$ \\
\hline I: Bus-cycle & 0.0284 & 0.0052 & 0.384 & 1.834 \\
\hline $\begin{array}{l}\text { II: Long-run } \\
\text { (symmetric) }\end{array}$ & 0.0384 & -0.0024 & 0.127 & 0.127 \\
\hline $\begin{array}{l}\text { III: Long-run } \\
\text { (asymmetric) }\end{array}$ & 0.0316 & -0.0126 & 0.085 & 0.191 \\
\hline$\delta \quad \gamma$ & $\psi$ & $\sigma_{c}$ & $\sigma_{e}$ & $\sigma_{d}$ \\
\hline $0.02 \quad 10$ & 1.5 & 0.015 & 0.1 & 0.1 \\
\hline
\end{tabular}

subject to the final condition $B(\pi, T ; T) \equiv 1$, and two boundary conditions at $\pi \in \partial(0,1)$ of the form

$$
\begin{aligned}
& 0=\partial_{t} B(0, t ; T)+\beta_{1}(0) \partial_{\pi} B(0, t ; T)+\beta_{0}(0) B(0, t ; T) \\
& 0=\partial_{t} B(1, t ; T)+\beta_{1}(1) \partial_{\pi} B(1, t ; T)+\beta_{0}(1) B(1, t ; T)
\end{aligned}
$$

where the functions $\beta_{i}(\pi), i=0,1,2$ are displayed in Appendix.

(iii) The conditional expected excess return on the bond is given by

$$
\mu_{B}(t ; T)-r(\pi(t))=(1-\alpha) \widetilde{\boldsymbol{\Sigma}}_{C}^{\prime} \widetilde{\boldsymbol{\Sigma}}_{B}(t ; T)+(1-\alpha / \rho) \widetilde{\boldsymbol{\Sigma}}_{\pi}^{\prime}(\pi(t)) \widetilde{\boldsymbol{\Sigma}}_{B}(t ; T) \partial_{\pi} \log \Psi(\pi(t))
$$

(iv) The equilibrium conditional volatility of the bond is given by

$$
\widetilde{\mathbf{\Sigma}}_{B}(t ; T)=\widetilde{\mathbf{\Sigma}}_{\pi}(\pi(t)) \partial_{\pi} \log B(t ; T)
$$

Proof. See Appendix.

\section{Quantitative Analysis}

\subsection{Calibration}

We consider 3 different calibrations of the Markov chain that drives the dynamics of consumption growth rates. Results of the calibration are reported in Table 1 ,

The first calibration is from Cagetti, Hansen, Sargent, and Williams (2002), who estimate the hidden Markov chain with the cumulative Solow residual from Citibase using maximum likelihood. According to their estimates, the mean duration is 2.61 years for the high-growth state, 0.55 year for the low-growth state, which suggest that the Markov chain dynamics are at business-cycle 
Table 2: Moments of the Posterior Distribution

\begin{tabular}{cccccc}
\hline & Mean & Median & Mode & Std & $95 \%$ Conf. Interval \\
\hline $\bar{\theta}$ & 0.5684 & 0.6125 & 0.5820 & 0.1600 & {$[0.4996,0.9779]$} \\
$\underline{\theta}$ & 0.1034 & 0.1195 & 0.3186 & 0.2417 & {$[-0.4053,0.4512]$} \\
$\sigma$ & 0.4659 & 0.4652 & 0.4626 & 0.0272 & {$[0.4148,0.5211]$} \\
$p_{11}$ & 0.9232 & 0.9509 & 1.0000 & 0.0896 & {$[0.6866,0.9960]$} \\
& & & & & \\
$p_{22}$ & 0.8236 & 0.8586 & 1.0000 & 0.1481 & {$[0.4203,0.9930]$} \\
\hline
\end{tabular}

NOTE - These results are based on 40,000,000 simulations, the first 20,000,000 of which were discarded to ensure the convergence of the Gibbs sampler. We do impose the necessary constraint that the (finite-interval) transition probability matrix $P$ over a quarter actually has an infinitesimal generator. As there are only 2 states, this amounts to the condition that the determinant $\operatorname{det}(P)>0$ and $\operatorname{trace}(P)>1$. Sample period 1947:Q1 - 2007:Q2.

frequency.

The second calibration is based on the long-run risk model of Bansal and Yaron (2004). We use a two-state Markov chain to approximate the discrete-time AR(1) process for expected growth rates in the model with homoskedastic consumption growth (Case I of BY). These numbers are calibrated to match the sample moments of annual consumption data from 1929 to 1998. The approximation method is based on the quadrature method of Tauchen and Hussey (1991). Chen (2007) explains the procedure in detail. Because the Bansal and Yaron model of consumption growth is Gaussian, a two-state approximation gives a symmetric Markov chain. This implies that the economy spends the same aount of time in the high-growth and low-growth state. The resulting mean duration is 7.86 years for both states, suggesting that the Markov chain captures consumption dynamics at much lower frequency than the business cycle.

The third calibration adds asymmetry to the Markov chain. In the case of the business cycle calibration, the good state has a duration roughly 4 times as long as the bad state. We introduce similar feature into the long-run risk calibration by multiplying $\lambda$ with a factor of $1 / 1.5, \mu$ with a factor of 1.5 , but also adjust $(\bar{\theta}, \underline{\theta})$ so that the steady-state distribution of $\theta$ has the same mean and variance as the BY model.

To see whether these calibrations are reasonable, we directly estimate a two-state Hidden Markov Model (HMM) using the Gibbs sampler. Our consumption data span the quarters from 

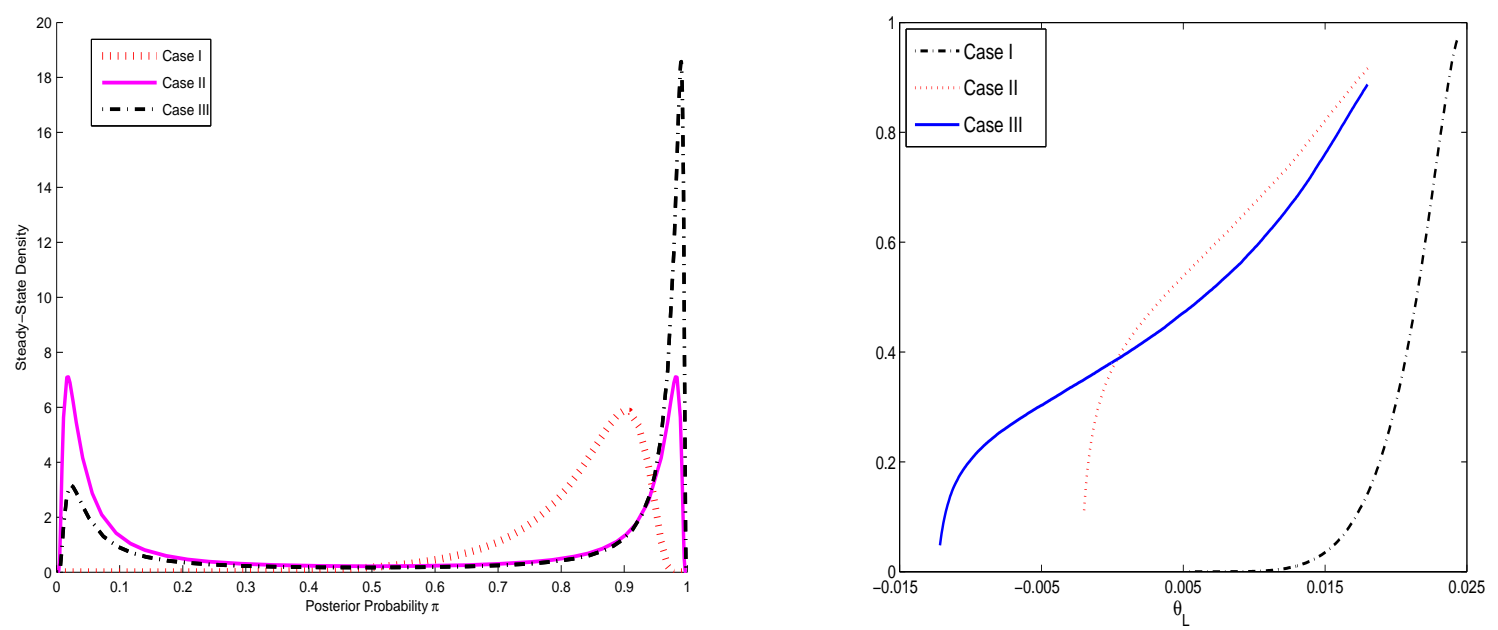

Figure 2: Left Panel: The steady-state distribution of the posterior belief of good state. Right Panel: Probability of posterior belief dropping below threshold $\theta_{L}$ at least once in a 100-year period.

1947:Q1 up to 2007:Q2, and consist of the sum of nondurable goods and services. They are converted to per-capita values by dividing by the population size of the U.S. Recall that the model stipulates the dynamics of the consumption growth as

$$
\frac{d C}{C}=\theta d t+\sigma_{c} d Z^{c}
$$

where $\theta$ changes between 2 states according to a continuous-time Markov chain. To take this model to data, we discretize the above equation as follows

$$
\frac{\Delta C_{t}}{C_{t}}=\theta\left(s_{t}\right)+\sigma_{c} \varepsilon_{t}^{c}
$$

where $\varepsilon_{t} \sim$ i.i.d. $\mathbb{N}(0,1)$, and $s_{t}$ is a latent variable following a hidden Markov chain (Hamilton (1994)). We cast the estimation in the Bayesian framework using multi-move Gibbs sampler.

Table 2 reports the results of the estimation. The numbers are quarterly, and growth rates and volatility are in percentage. Not surprisingly, the state and transition probabilities of the hidden Markov chain are estimated very imprecisely, while the volatility of consumption growth is estimated with high precision. One can verify that the parameters for all three calibrations in Table 1 fall within the 95\% confidence interval of the Gibbs Sampler estimation.

The steady-state distributions of the posterior belief of good state in the three calibrations are reported in the left panel of Figure 2. The distribution is unimodal in Case I, but bimodal in the two cases with long-run risk. It is more likely for investors to have high local uncertainty ( $\pi$ close to 0.5) in Case I, while in Case II and III, investors are rarely uncertain of the state they are in. In Case III, most of the time investors believe that they are in a good state. Thus, getting to the bad 

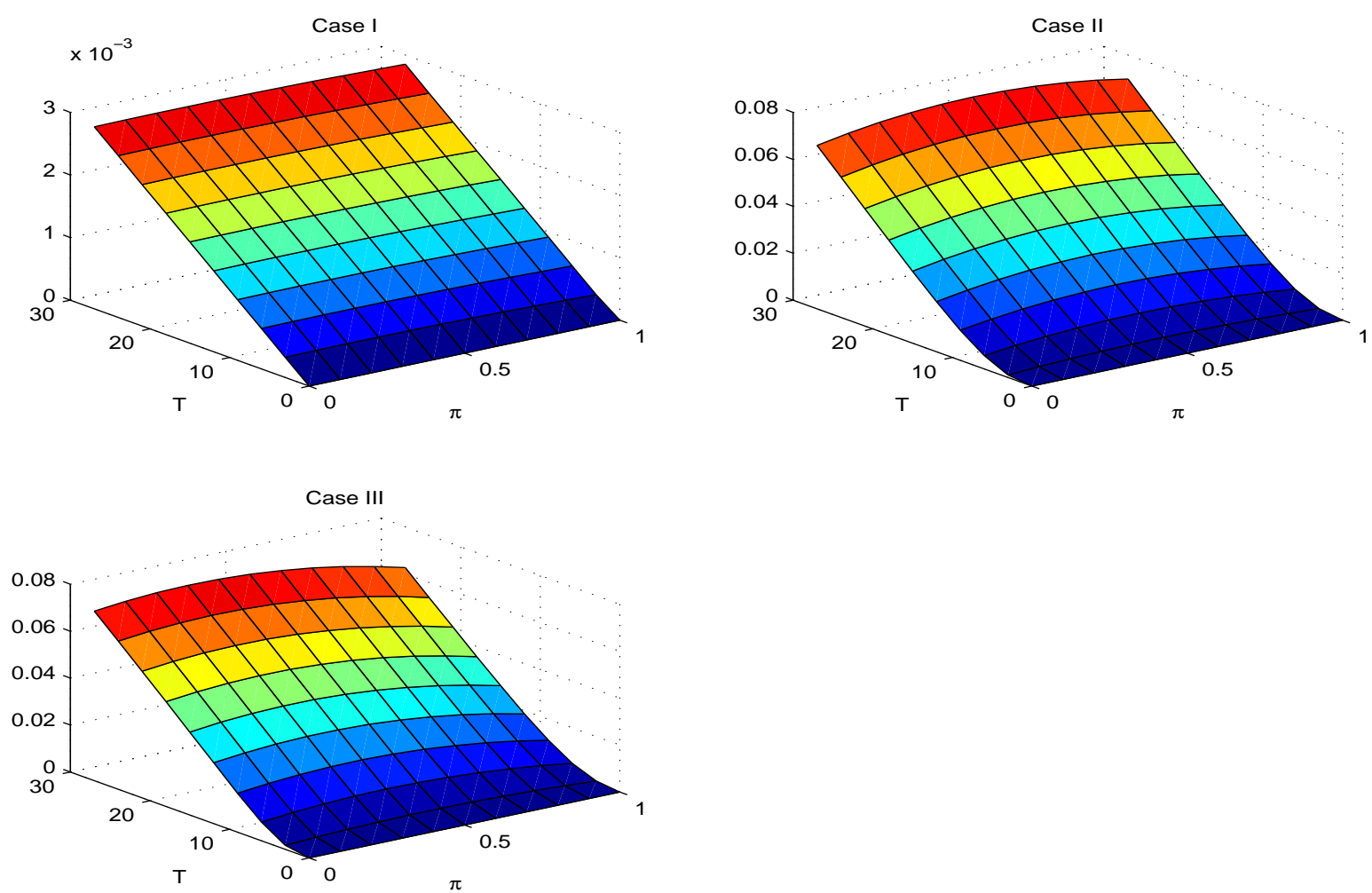

Figure 3: Long-Run Uncertainty of Growth Rates

state is a "rare event" in this case. The right panel of Figure 2 investigates this property formally by reporting the probability that the posterior belief drops below a given threshold at least once in a random 100-year sample.

In Section 2.2, we define long-run uncertainty $V(t, T)$ as the uncertainty about accumulative growth rates from time $t$ to $T$ in the future. Figure 3 plots $V(t, T)$ for the three cases of calibration. In each case, $V(t, T)$ grows with the horizon $T$, but the speed of growth is much higher when the states are persistent, leading to long-run uncertainty that is an order of magnitude larger. These results suggest that, when there is a long-run component in the growth rate, small local uncertainty about the growth rate gets amplified into large long-run uncertainty. This is the key source of high uncertainty premium in the model. Moreover, $V(t, T)$ is symmetric and peaks at $\pi=0.5$ in Case II (when the Markov chain is symmetric), but skewed in Case III.

\subsection{Consumption Claims and Levered Stock}

Figure 4 plots the wealth-consumption ratio, conditional risk premium, Sharpe ratio, and conditional return volatility of aggregate consumption claim for three sets of calibrations. In the case of business cycle frequency dynamics of consumption growth, these quantities are only weakly dependent on the posterior probability. The Sharpe ratio increases ranges from 0.1 when $p i$ is close 

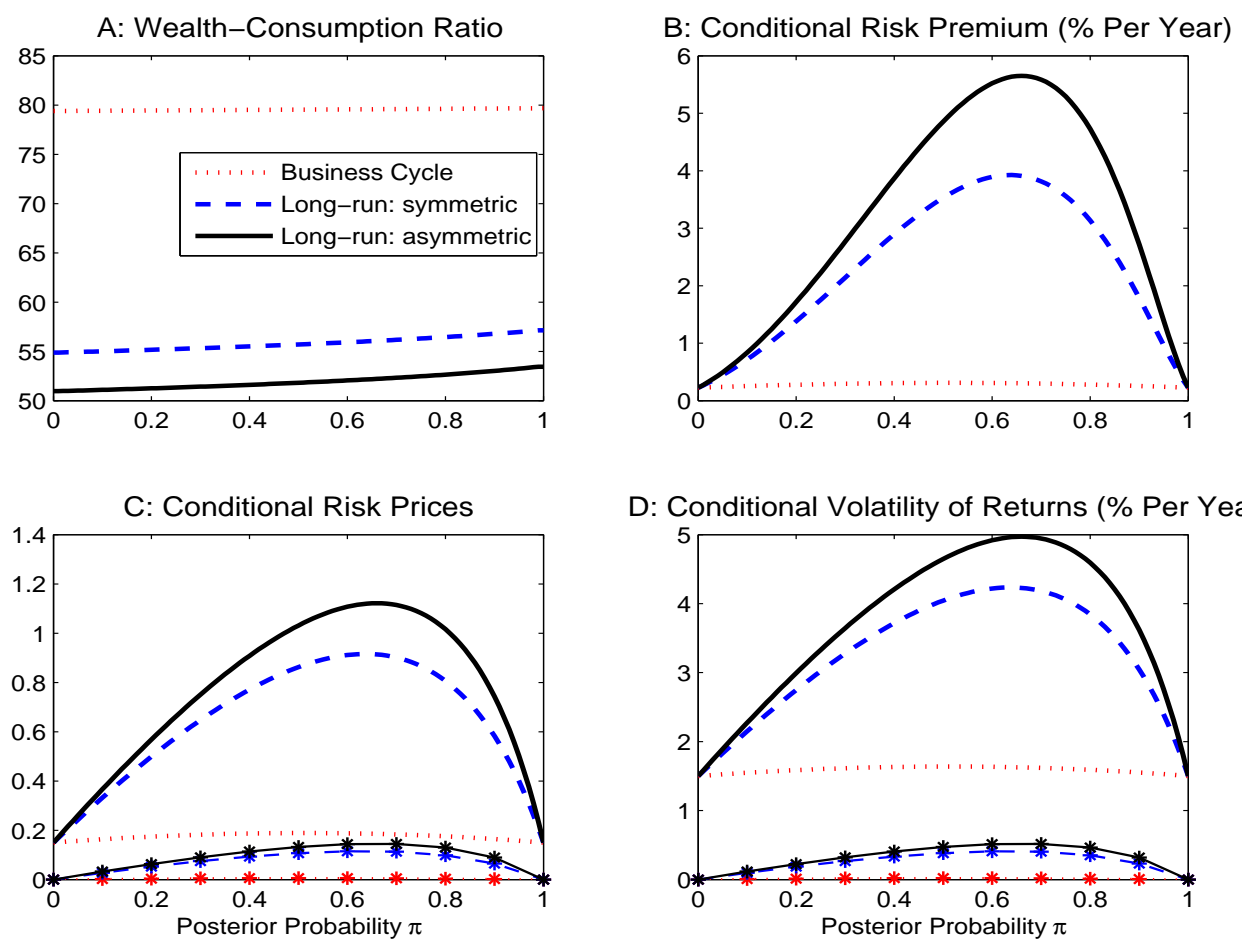

Figure 4: Wealth-consumption ratio, conditional risk premium, Sharpe ratio, and conditional return volatility of aggregate consumption claim for three calibrations. Panel $\mathrm{C}$ and $\mathrm{D}$ show the risk prices and volatility for consumption shocks and signal shocks (marked with stars) separately.

to 0 or 1 , to the peak of about 0.15 when $\pi$ is around 0.5 , which is similar in magnitude to the risk prices in Hansen (2007).

Moving to the case of long-run dynamics, wealth-consumption ratio drops significantly, while risk premium, Sharpe ratio and conditional return volatility become hump-shaped and left-skewed. Compared to the business-cycle case, the risk premium is much larger and has significant variation over different values of $\pi$.

When we introduce asymmetry between the two states but keeping the mean and variance of the steady-state distribution of the expected growth fixed, we are increasing the skewness of the distribution. In this case, wealth-consumption ratio drops further, while risk premium, Sharpe ratio and conditional return volatility are become higher and further skewed. The peak of the risk premium for consumption claims is now a sizable $6 \%$ per year, compared to $0.2 \%$ in the first case.

Next, in Figure 5, we investigate the effects of risk aversion and EIS on prices and returns using the Markov chain parameters from Case III of the calibration. We consider the combinations of two levels of relative risk aversion and EIS. Since EIS is larger than 1 in all cases, wealth-consumption ratio is increasing in the posterior probability. Clearly, higher risk aversion increases the Sharpe ratio, volatility, and risk premium. EIS appears to have negligible effects on the Sharpe ratio, but 

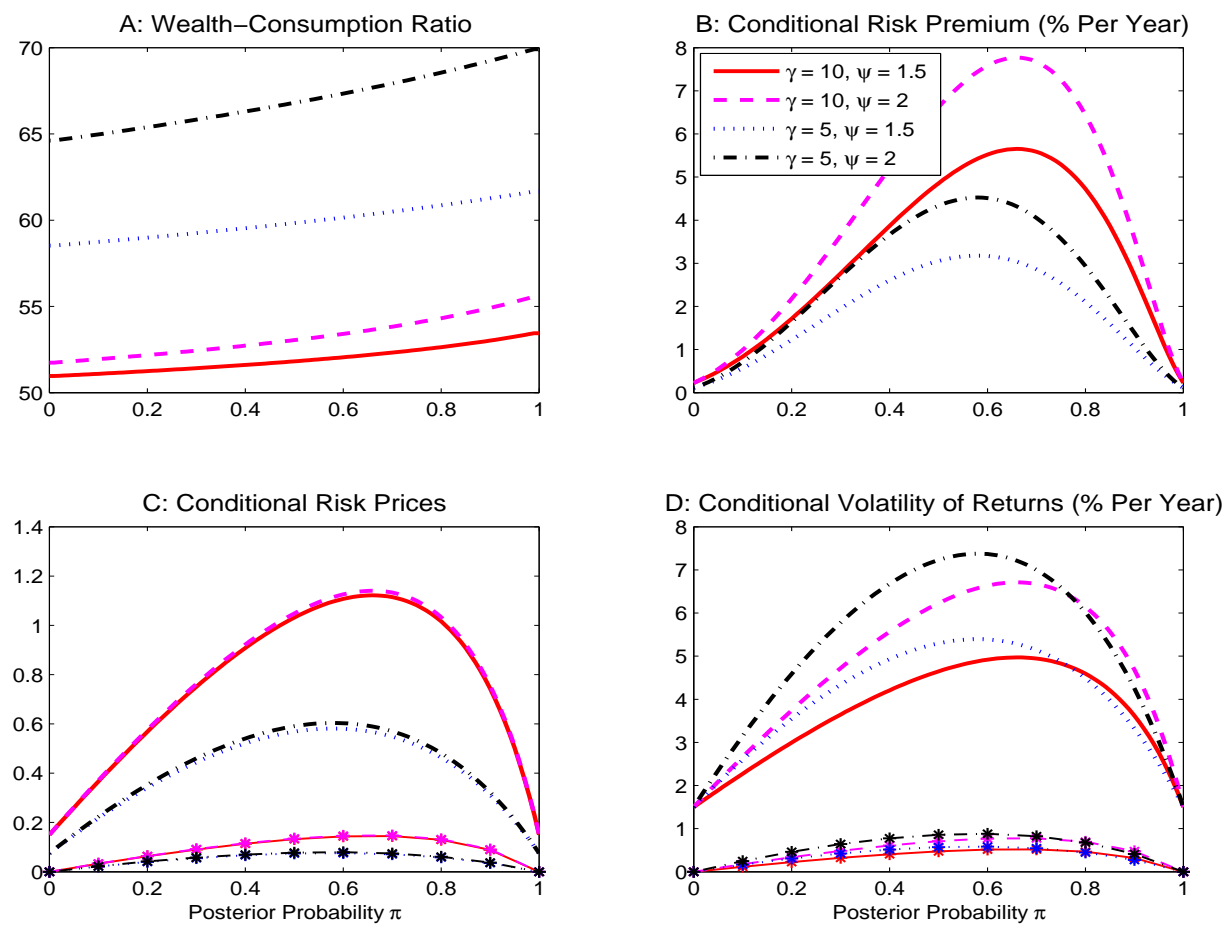

Figure 5: Effects of risk aversion and elasticity of intertemporal substitution on prices and returns. Panel $\mathrm{C}$ and $\mathrm{D}$ show the risk prices and volatility for consumption shocks and signal shocks (marked with stars) separately.

higher EIS does raise the volatility of returns, which in turn raises the risk premium.

So far we have been focusing on cases with $\psi>1$ and $\gamma>1 / \psi$. In all these cases, wealthconsumption ratio increases with the posterior probability of good state $\pi$, and there is a positive premium for uncertainty risk. Figure 6 explores other different scenarios. For comparison, we consider two sets of calibrations for the EIS: $\psi=0.2$ and $\psi=2$. For each value of $\psi$, we also consider three different values of $\gamma$ that imply three different preferences for the timing of resolution of uncertainty.

When EIS is less than 1, wealth-consumption ratio decreases with $\pi$, and returns are negatively correlated with consumption shocks (as shown with negative sign for the conditional volatility). These findings are consistent with Veronesi (2000). In particular, the Sharpe ratio in the power utility case is flat, while the risk premium becomes negative due to the negative correlation between returns and consumption shocks. When $\psi<1$ and $\gamma>1 / \psi$ (solid line), there is a positive risk price for uncertainty. However, the conditional risk premium is negative, again because of the negative correlation between returns and consumption shocks.

Finally, when $\psi<1$ but $\gamma<1 / \psi$ (dotted line), agents prefer later resolution of uncertainty, which leads to an uncertainty discount (negative risk prices). However, because of the negative 

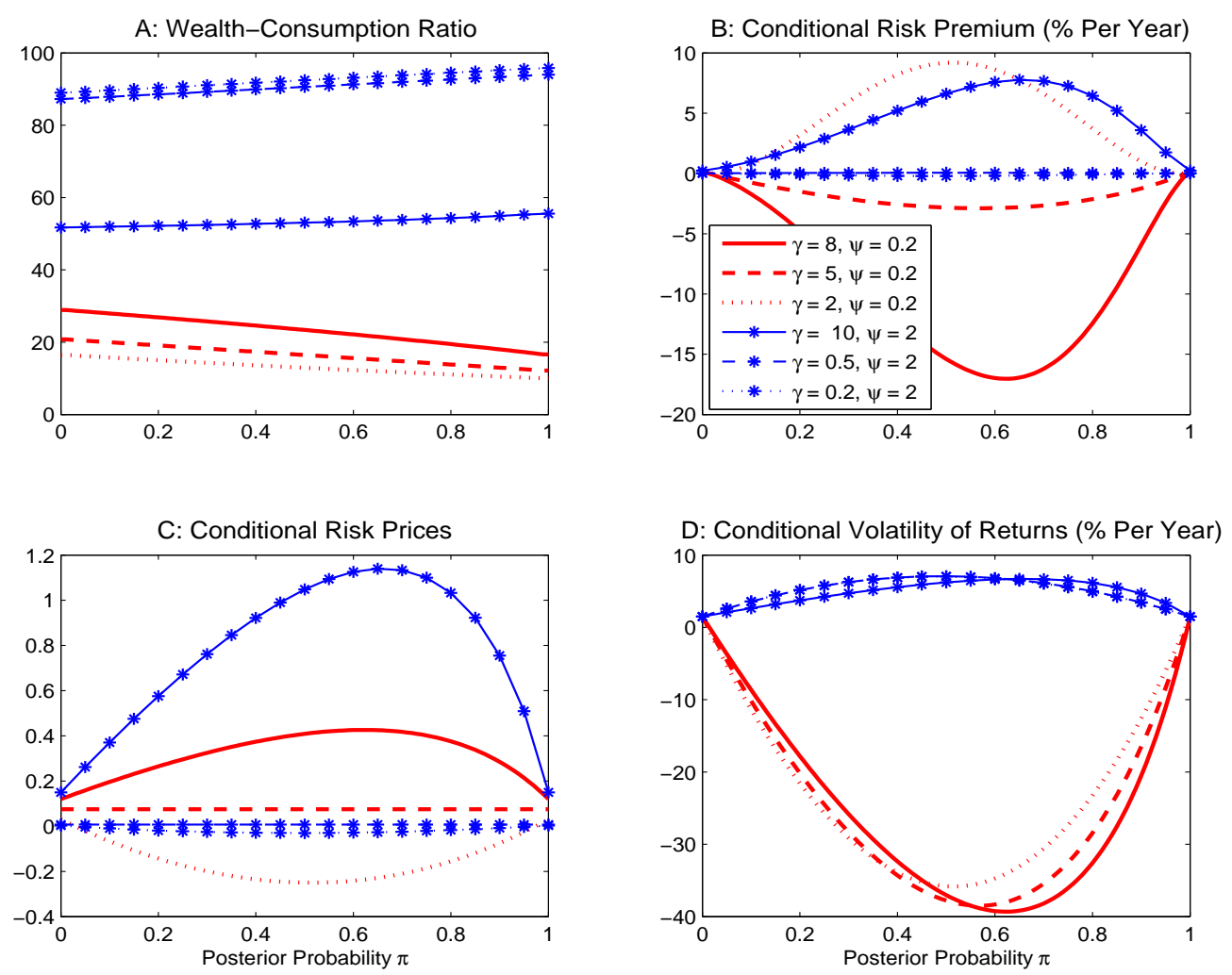

Figure 6: Effects of preferences for resolution of uncertainty on prices and returns. Panel C and D show the risk prices and volatility for consumption shocks and signal shocks (marked with stars) separately.

correlation between return and consumption shocks, this actually leads to a positive and sizeable risk premium. These different scenarios are worthy of further investigations.

Finally, in Figure 7, we study how consumption volatility affects prices and returns. When consumption volatility is relatively large, e.g. in the range of $2 \%$ to $4 \%$ per year, higher consumption volatility leads to higher volatility, Sharpe ratio, and risk premium. Surprisingly, when consumption volatility becomes sufficiently low, a decrease in consumption volatility actually increases volatility and returns.

The intuition for this result is as follows. Because when volatility of consumption becomes smaller, the signals about the hidden growth rate becomes more precise. As a result, agents will respond to consumption shocks more strongly, leading to larger volatility in the posterior probability $\pi$, which in turn raises the risk price for uncertainty, as well as the volatility of returns. The two effects combine to lead to higher risk premium.

Finally, the levered stock, claim on the dividend process, generates stronger variation in the price-dividend ratio, volatility, and risk premia. Results are reported in Figure 8. 

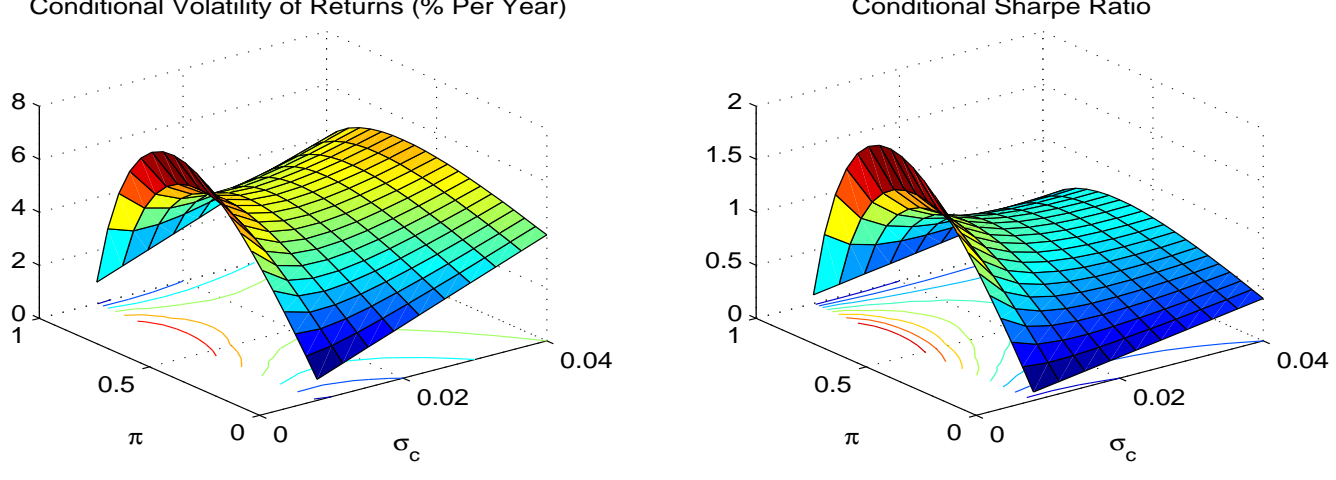

Conditional Risk Premium (\% Per Year)

Riskfree Rate (\% Per Year)
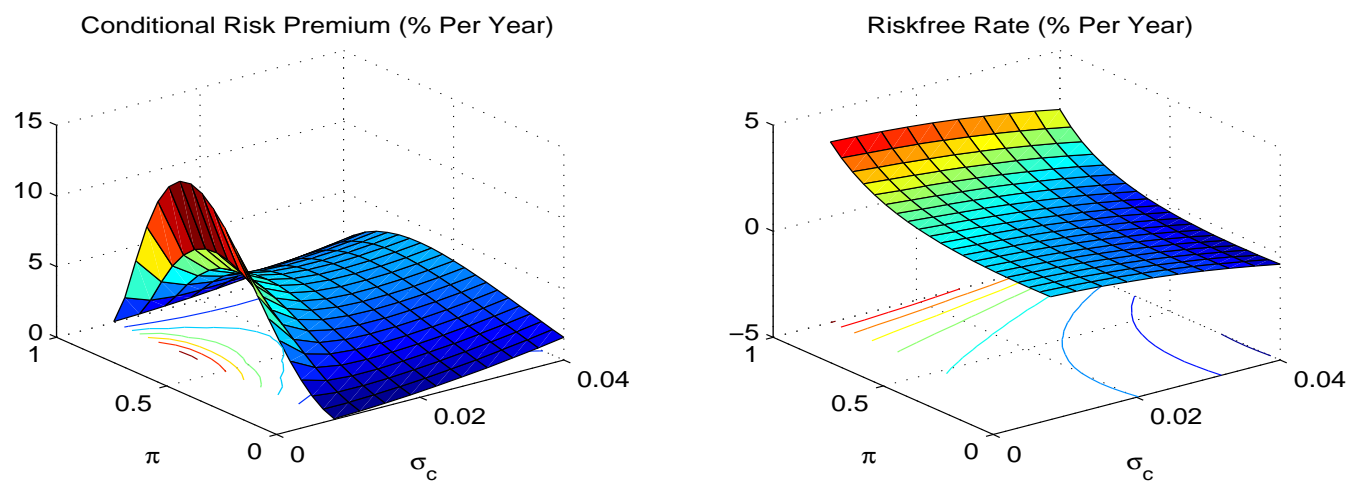

Figure 7: Effects of consumption volatility on prices and returns

\subsection{Stochastic Volatility, Uncertainty Premium, and Predictability in Stock Returns}

If the price-dividend ratio $S_{t} / D_{t}$ is a constant, the only reason that returns vary is that the real dividend growth rate varies. This is reflected in the term $\sigma_{D}$ in the Proposition. The additional term is $v(\pi) \Psi^{\prime}(\pi) / P s i(\pi)$, and reflects the contribution of a time-varying price-dividend ratio. Note that this term tends to maximized when the uncertainty about the true state of the economy is highest, that is, $\pi \approx 1 / 2$, the intuition being that during periods of high uncertainty, each new piece of information receives a large weight in the updating process of an posterior distribution 4 . But investors are cognizant that their expectations of future dividend growth will shift around a lot in times of uncertainty and their desire to hedge against their own uncertainty affects the equilibrium prices and hence returns. Due to investors' desire to hedge against the changes in their own uncertainty, periods of a high uncertainty are naturally times of high expected returns to compensate for high return volatility. It is therefore not surprising to find that the expected returns are time-varying.

\footnotetext{
${ }^{4}$ That this is so may be seen by investigating the functional form of $v(\pi)$, which is the variance of $d \pi$.
} 

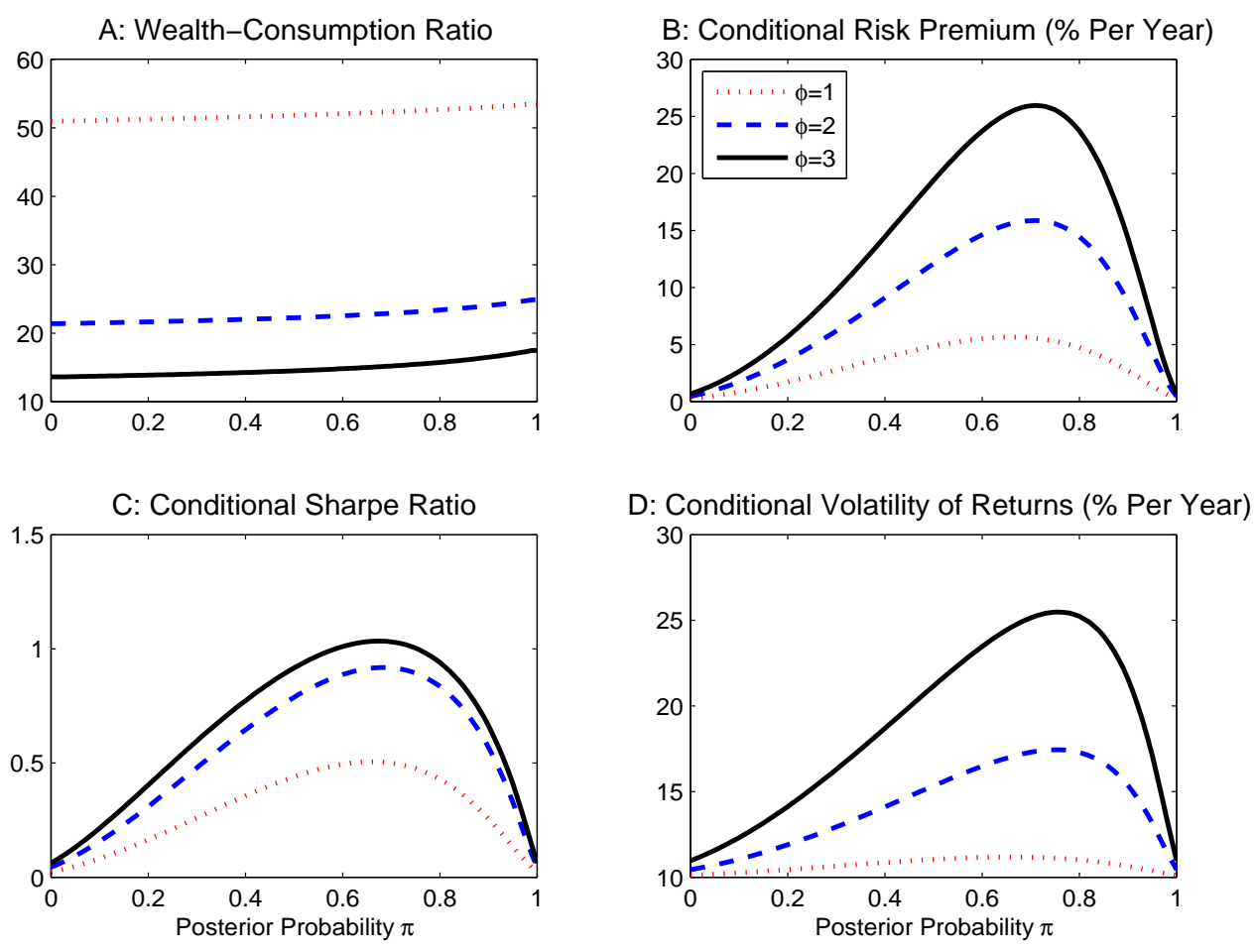

Figure 8: Prices and returns of levered stocks

Table ?? reports the bootstrapped predictability regressions for the scenarios that we consider. There are 100,000 bootstrap experiments performed, each time obtaining 100 non-overlapping annual time series. As may be observed, there is a huge sampling uncertainty as to the magnitude and sign of the regression slope on the (log) dividend yield. That makes a challenge to try to match empirical studies. Our focus, instead, is on the population moments.

What is important for predictability is the asymmetry of the states for consumption growth, with the bad state being less persistent. That corresponds to scenarious 1, 3 and 4, where, except case 3 due to a "too-high" RRA coefficient, we obtain the correct signs for the regression slopes. As Case 2 features symmetric states, we obtain a wrong sign for predictability. We conjecture and plan to imbedd in the paper in the near future, is a model that displays both both long-run risk and at the same time matches the business cycle dynamics. Such a model calls for a 4-state model for the expected consumption growth rate.

Figure 9 explains the source of predictability. On the left side, we simulate the process of $\pi$ and compute the correlation between posterior mean and uncertainty about the hidden growth rate. This correlation is significantly negative for both Case I and III calibrations, implying that most of the time a drop in expected growth rate comes with a rise in uncertainty. Because of the uncertainty premium, we can infer from this negative correlation that negative consumption shocks, which leads agents to lower their belief of $\pi$, will tend to increase the instantaneous expected 

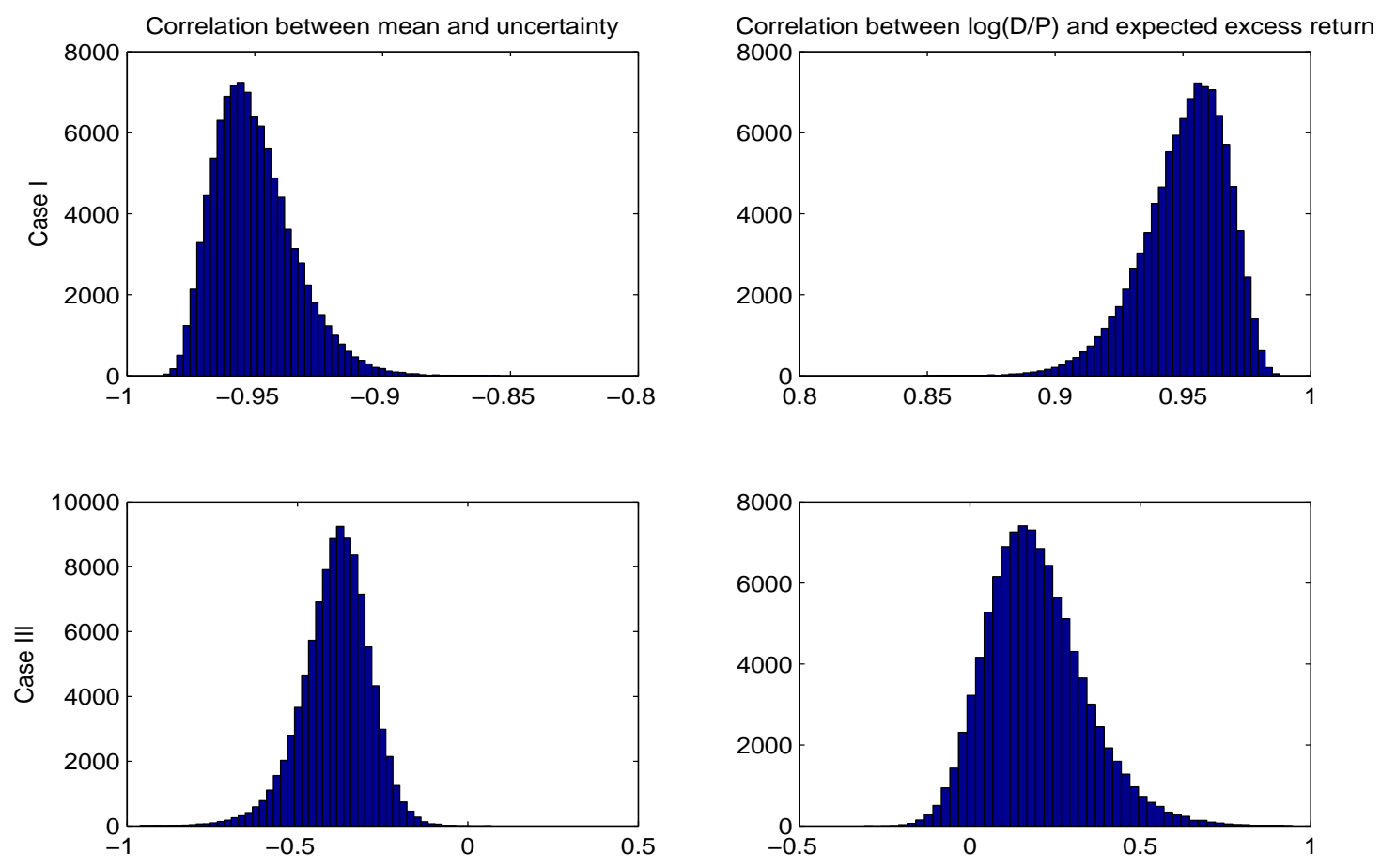

Figure 9: The distribution of the correlation between mean and uncertainty about the growth rate, and the correlation between log dividend price ratio and instantaneous expected excess return of the stock

excess return. This relation shows up on the right panel of the figure. To check whether the model generates similar return predictability as in the data (e.g., Cochrane (2007)), we also report the distribution of return-forecasting coefficient through Monte Carlo simulation in Figure 10.

\subsection{Real Term Structure}

Figure (11) displays the expected excess bond return and conditional volatility surfaces, for the calibrations that we consider. In all cases, the expected excess return is negative. Real zero-coupon bonds are a hedge against the long-run consumption uncertainty. In equilibrium, they covary negatively with consumption growth and hence command a negative risk premium. This fact is a direct consequence of a rise in precautionary saving demand in times of uncertainty. It is to be observed that as the persistence of the underlying hidden Markov chain increases, the expected excess returns rise (in absolute value) and become more skewed, attaining their maximum at $\pi$ greater than $1 / 2$. The conditional volatility also rises with the persistence of the states, attaining a hefty $5 \%$ in Case III calibration.

Figures 12 portray the equilibrium real yield curves. Their behavior is to a large extent dependent on the asymmetry of the good and bad state, and the persistence of the hidden Markov chain. 
Table 3: Unconditional Population Annualized Moments: Equity Market

\begin{tabular}{|c|c|c|c|c|}
\hline & \multicolumn{4}{|c|}{ Wealth-Consumption Ratio $W / C$} \\
\hline & Case I & Case II & Case III $(\gamma=10)$ & Case III $(\gamma=5)$ \\
\hline Mean Ratio & 415.23 & 126.97 & 109.93 & 154.17 \\
\hline AR1 of Ratio & 0.11 & 0.77 & 0.75 & 0.76 \\
\hline Mean Excess Return (\%) & 0.27 & 1.21 & 1.52 & 0.76 \\
\hline Std of Excess Return (\%) & 1.53 & 2.44 & 2.45 & 2.49 \\
\hline AR1 of Excess Return & 0.02 & 0.12 & 0.06 & 0.01 \\
\hline Average Sharpe Ratio & 0.17 & 0.41 & 0.47 & 0.23 \\
\hline Min Sharpe Ratio & 0.15 & 0.15 & 0.15 & 0.08 \\
\hline \multirow[t]{3}{*}{ Max Sharpe Ratio } & 0.19 & 0.93 & 1.15 & 0.60 \\
\hline & \multicolumn{4}{|c|}{ Price-Dividend Ratio $S / D$} \\
\hline & Case I & Case II & Case III $(\gamma=10)$ & Case III $(\gamma=5)$ \\
\hline Mean Ratio & 258.71 & 59.24 & 46.21 & 75.33 \\
\hline AR1 of Ratio & 0.11 & 0.76 & 0.74 & 0.75 \\
\hline Mean Excess Return (\%) & 0.52 & 3.87 & 5.58 & 3.30 \\
\hline Std of Excess Return (\%) & 10.28 & 11.20 & 11.31 & 11.42 \\
\hline AR1 of Excess Return & 0.00 & 0.05 & 0.04 & 0.00 \\
\hline Average Sharpe Ratio & 0.05 & 0.33 & 0.45 & 0.25 \\
\hline Min Sharpe Ratio & 0.03 & 0.03 & 0.03 & 0.02 \\
\hline \multirow[t]{3}{*}{ Max Sharpe Ratio } & 0.06 & 1.09 & 1.54 & 1.04 \\
\hline & \multicolumn{4}{|c|}{ Real Risk-Free Rate $r^{f}$} \\
\hline & Case I & Case II & Case III $(\gamma=10)$ & Case III $(\gamma=5)$ \\
\hline Mean $(\%)$ & 2.41 & 1.39 & 1.20 & 1.71 \\
\hline $\operatorname{Std}(\%)$ & 0.18 & 1.12 & 1.42 & 1.28 \\
\hline AR1 & 0.11 & 0.56 & 0.49 & 0.66 \\
\hline
\end{tabular}

$\mathbb{N O T E}$ - The table reports the annualized unconditional moments of the wealth portfolio return, of the levered consumption claim return, of the real risk-free rate, and the unconditional means and $\mathrm{AR}(1)$ coefficients of the wealth-consumption ratio $W / C$ and the price-dividend ratio $S / D$. Based on 100,000 years of simulated data. 


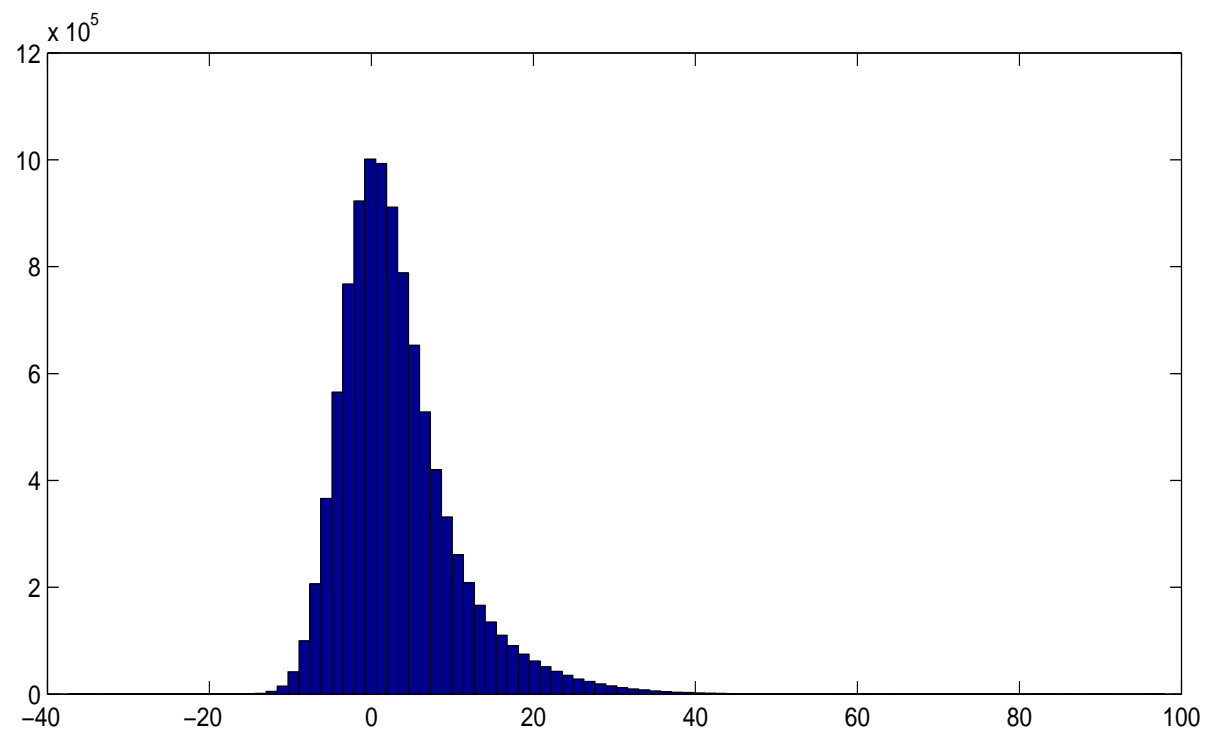

Figure 10: Bootstrap of Return Predictability Regression. We simulate a 50-year sample and run the following regression with annual data: $R_{t+1}=a+b \frac{D_{t}}{P_{t}}+\epsilon_{t+1}$. The histogram reports the distribution of coefficient $b$ from 1 million simulations.

Calibration I features asymmetric states with a fairly low persistence; the relevant intensities are $\lambda=0.384$ and $\mu=1.834$, with the steady-state probability of the high consumption state being $\pi^{s}=1.834 /(1.834+0.384)=0.827$. The real yield curves are on average flat, with the most action happening at the very short end of the term structure. This may be also seen in Table 4.4.1. The average term structure of one-year forward rates for 1- to 5 - years is flat at about $3.40 \%$ p.a, with practically zero volatility. The forward-spot spread fluctuates predominantly due to a variation in the one-year spot rate. Although expected term premia vary over time for very short maturities (less than one year), as dictated by the previous lemma, the term structure of expected term premia for horizons 1 to 5 years is practically flat, with the concomitant population volatility only about 8 basis points. The first-order autoregressive coefficient is close to zero, and we do not get much action in terms of predictability at horizons over one year.

Calibration II corresponds to a (still) symmetric but much more persistent Markov chain driving the consumption growth, and we obtain richer dynamics for the longer-term yields. The steadystate probability of the good state is $1 / 2$, and the real yield curves are on average downward sloping as the forward rates decline with respect to the bond maturities (see Table 4.4.1, calibration II), and forward spot spreads are negative on average. The real spot and forward rates are much more persistent, $\mathrm{AR}(1)$ on the order of 0.7 , though not as much as their nominal empirical counterparts (Table 2, Fama Bliss). We see that as the persistence of the Markov chain rises, we get more action in the middle of the yield curve (years 1-5) where one-year forward rate volatility jumps up from zero to more than 10 basis points. The term structure of expected term premia is negative (real 

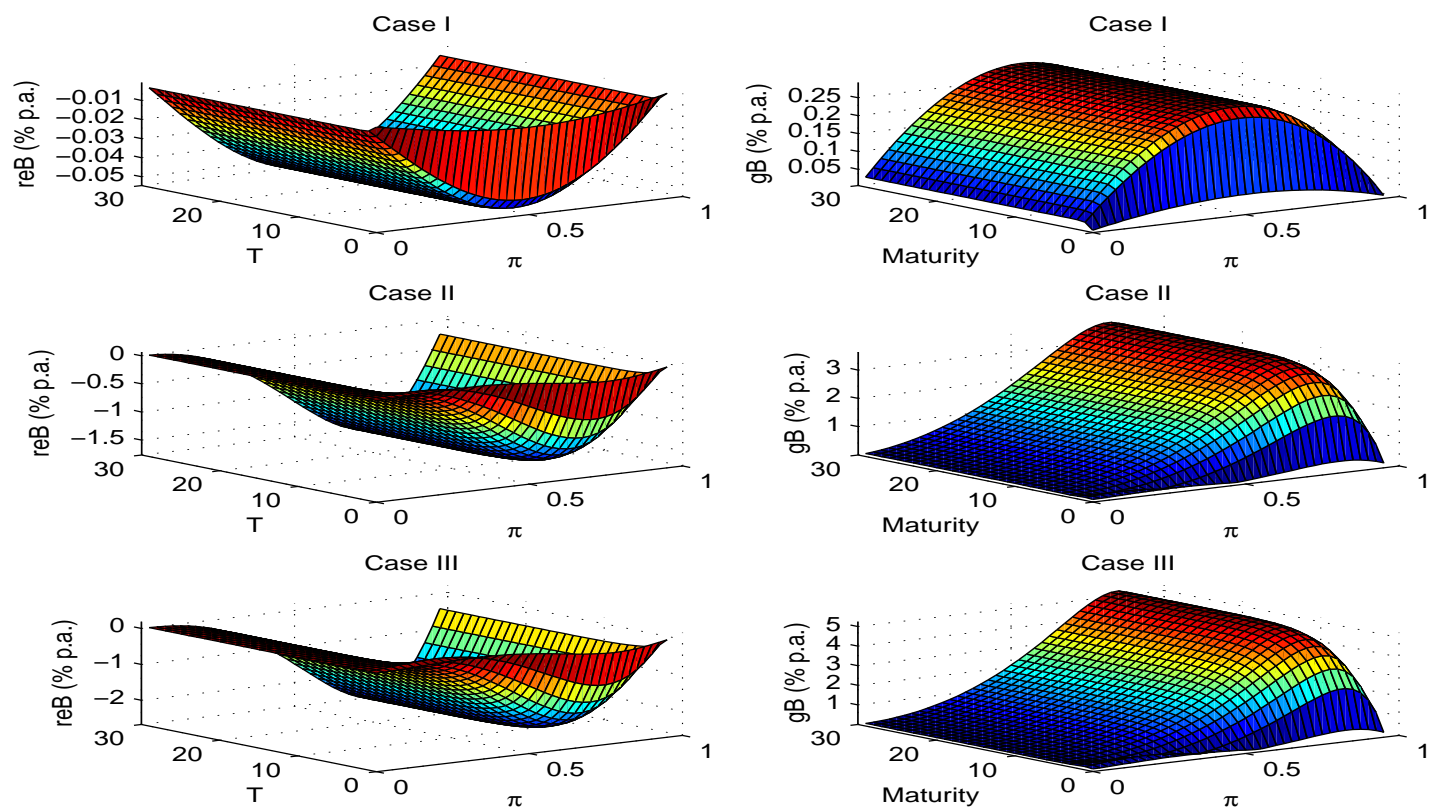

Figure 11: Expected excess bond return and conditional volatility surface

bonds are consumption hedges) and declining with maturity, altough their persistence by far does not match their empirical nominal counterparts. Our investigation focuses on real bonds and hence the discrepancy might be attributed to a very persistent inflation risk in the expected term premia of nominal bonds.

In order to match the predictability of excess equity returns, we do need asymmetric states; the good state must have higher duration than the bad state. This brings us to calibration III and IV which differ only in the coefficient of risk aversion $\gamma$; it is either 10 (III) or 5 (IV). We see a steeply declining term structure of expected term premia on bonds. The best hedge against economic uncertainty are long-term bonds; they covary negatively with consumption growth rate and hence command the lowest expected excess return. In addition, expected term premia are comparatively quite variable, with their population volatility rising from 69 basis points at the horizon of one year up to more than 100 basis points for year 5 . In both cases, real yield curves are downward sloping, as forward-spot spreads are negative and growing with maturity. Cases III and IV differ in the calibration of $\gamma$. As gamma declines from 10 down to 5, we can almost match the persistence of forward-spot spread though certainly not their volatility which is 2-3 times lower than for nominal forward-spot spreads.

In all cases, the term structure of forward rate volatilities is downward sloping whereas the term structure of expected returns volatilities is upward sloping. 

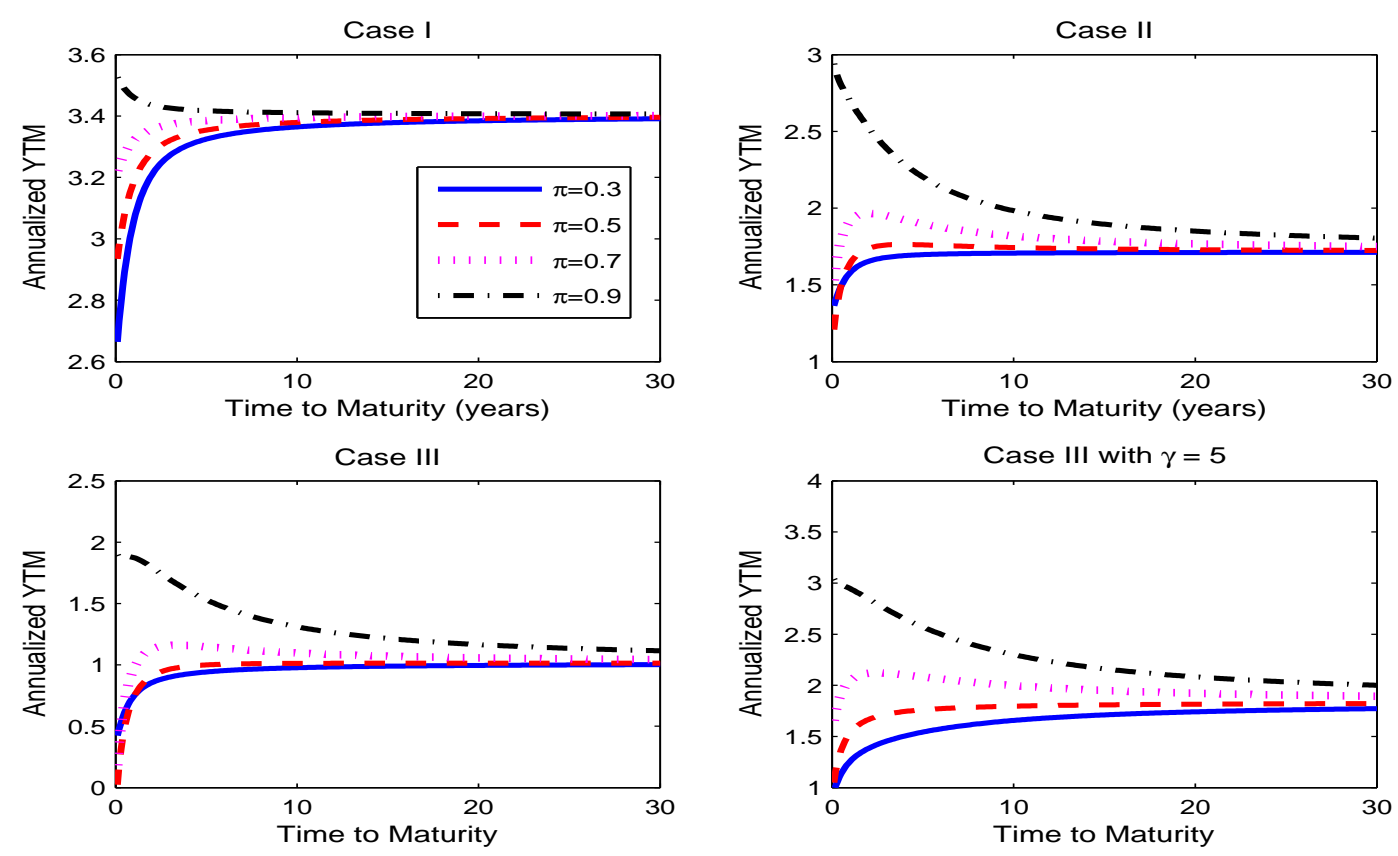

Figure 12: Equilibrium real yield curve

\subsubsection{Fama-Bliss Regressions}

We denote the log-price at time $t$ of a zero-coupon bond with maturity $T$ as

$$
b(t ; T)=\log B(\pi(t), t ; T) .
$$

The annualized log-yield is then

$$
y(t ; T)=-\frac{1}{T} b(t ; T),
$$

and the $\log$ forward rate at time $t$ for loans between times $T$ and $T+1$ is

$$
f(t ; T)=b(t ; T)-b(t ; T+1)
$$

We reserve $y(t ; 1)$ for the real one-year spot rate, and call the forward rate in excess of the spot rate the forward-spot spread. In addition, we write the log holding-period return from buying at time $t$ a zero-coupon bond of maturity $T$ and selling it one year later as

$$
h p r(t+1 ; T)=b(t+1 ; T-1)-b(t ; T)
$$

We call the realized return in excess of of the spot rate the term premium. 
Simple manipulations of these definitions allows one to express the forward-spot spread as

$$
f(t ; T)-y(t ; 1)=-\mathbb{E}_{t}[h p r(t+1 ; T)-y(t ; 1)]+\mathbb{E}_{t}[(T+1) y(t ; T+1)-(T-1) y(t+1 ; T-1)]
$$

The forward-spot spread is a (noisy) measure of expected term premia. Fama and Bliss (1987), and Cochrane and Piazzesi (2005), perform simple regression tests of the information in forward rates about future expected returns on longer-term nominal bonds. They consist in regressing future term premia on the forward-spot spread. As these regressions appear to be a robust feature of the bond data, we quantitatively evaluate the ability of our model to fit the signs and magnitudes of the relevant regression coefficients.

Table 5 reports the simulations results. We perform 10,000 bootstrap experiments, each involving 40 years of non-overlapping annual data. We choose 40 years as it closely matches the length of sample periods used in empirical studies. We report the slopes, $R^{2}$ s and the $95 \%$ confidence intervals corresponding to the Fama-Bliss regression

$$
h p r(t+1 ; T)-y(t ; 1)=\beta_{0}+\beta_{1} \times[f(t ; T)-y(t ; 1)]+\varepsilon(t+1 ; T)
$$

For comparison, we also report the related population moments, obtained as simple averages of the simulated data over 100,000 years (Table 4.4.1).

As before, we consider four calibrations. The bootstrap results indicate that there is a huge sampling uncertainty as to the true population moments. The slopes commonly change signs and the $R^{2}$ s fluctuate between zero up to $15-20 \%$, making it a challenge to attempt to match empirical studies. Instead, we focus on matching the population moments. The reader, however, should be beware in that these studies analyze nominal bonds, not real ones, a point to which we return later.

Fama and Bliss (1987), Table 1, report their estimates from the regression (63) for the nominal bonds, sample period 1965-1985. Their all slopes come out positive and tend to rise with the horizon, along with their $R^{2} \mathrm{~s}$. As the forward-spot spread is variable, they conclude that the expected term premia on bonds with maturities of 1- to 5- years fluctuate over time. We know from the lemma above that expected term premia do vary over time, at least over the forward infinitesimal interval $(t ; t+d t)$. What the population slopes in Table 5 tell us that is that for the case I such a variation in expected returns is restricted to the very short end of the yield curve. The slopes come out economically insignificant -0.02 , and have the wrong sign, a problem which also occurs in case IV and deserves a closer analysis hereafter. Also, the average term-structure of forward rates is flat, and forward-spot rates volatilities are on the order of 7 basis points. And because the term structure of expected term premia volatilities is small too, there is nothing to predict as expected excess returns barely move. With respect to cases II and III, the slope coefficients have the correct, positive, sign, with $R^{2}$ s rising over time, and hence we conclude that we are able to qualitatively match the Fama-Bliss regressions results, conditional upon the assumption that such results would be upheld for real bonds as well. 
Table 4: Real Zero-Coupon Bond Market : Population Means, Standard Deviations and First-Order Autocorrelations

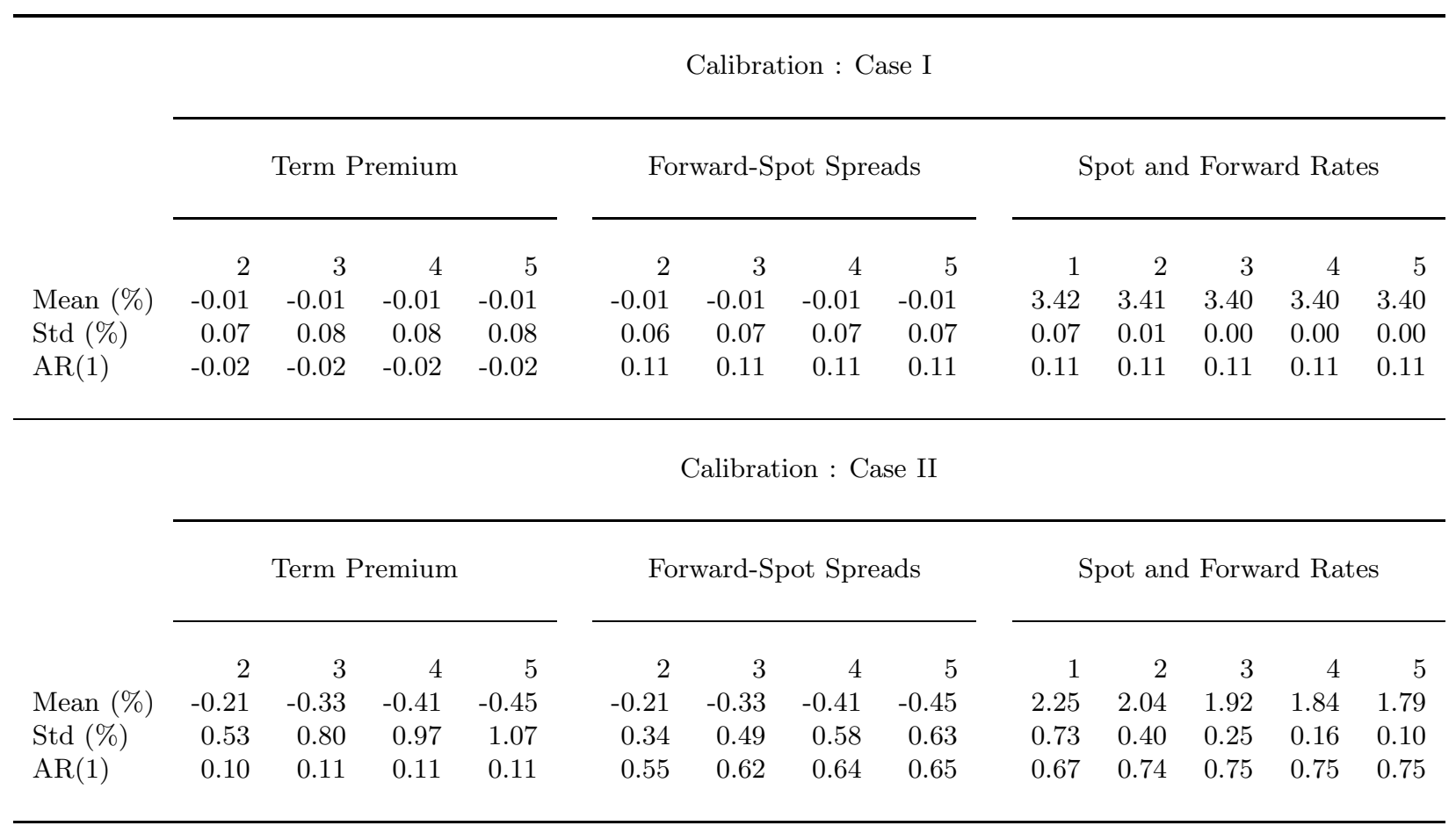

Calibration : Case III

\begin{tabular}{|c|c|c|c|c|c|c|c|c|c|c|c|c|c|}
\hline & \multicolumn{4}{|c|}{ Term Premium } & \multicolumn{4}{|c|}{ Forward-Spot Spreads } & \multicolumn{5}{|c|}{ Spot and Forward Rates } \\
\hline & 2 & 3 & 4 & 5 & 2 & 3 & 4 & 5 & 1 & 2 & 3 & 4 & 5 \\
\hline Mean (\%) & -0.32 & -0.52 & -0.65 & -0.74 & -0.32 & -0.52 & -0.65 & -0.74 & 1.94 & 1.62 & 1.42 & 1.29 & 1.20 \\
\hline $\operatorname{Std}(\%)$ & 0.65 & 0.99 & 1.21 & 1.36 & 0.38 & 0.54 & 0.65 & 0.72 & 0.86 & 0.50 & 0.33 & 0.22 & 0.15 \\
\hline $\operatorname{AR}(1)$ & 0.01 & 0.01 & 0.01 & 0.01 & 0.46 & 0.54 & 0.58 & 0.60 & 0.62 & 0.71 & 0.72 & 0.72 & 0.72 \\
\hline
\end{tabular}

Calibration : Case III with $\gamma=5$

\begin{tabular}{|c|c|c|c|c|c|c|c|c|c|c|c|c|}
\hline \multicolumn{4}{|c|}{ Term Premium } & \multicolumn{4}{|c|}{ Forward-Spot Spreads } & \multicolumn{5}{|c|}{ Spot and Forward Rates } \\
\hline 2 & 3 & 4 & 5 & 2 & 3 & 4 & 5 & 1 & 2 & 3 & 4 & 5 \\
\hline-0.17 & -0.31 & -0.42 & -0.50 & -0.17 & -0.31 & -0.42 & -0.50 & 2.60 & 2.43 & 2.29 & 2.19 & 2.10 \\
\hline 0.69 & 1.19 & 1.58 & 1.87 & 0.28 & 0.45 & 0.59 & 0.69 & 1.02 & 0.76 & 0.58 & 0.44 & 0.34 \\
\hline-0.04 & -0.03 & -0.03 & -0.03 & 0.61 & 0.68 & 0.70 & 0.71 & 0.73 & 0.75 & 0.75 & 0.75 & 0.75 \\
\hline
\end{tabular}

$\mathbb{N O T E}$ - Results are based on 100,0000 years of simulated data. 
Table 5: Term Premium Regressions

\begin{tabular}{|c|c|c|c|c|c|c|}
\hline \multirow[b]{3}{*}{ Horizon } & \multicolumn{6}{|c|}{ Calibration I } \\
\hline & \multicolumn{4}{|c|}{ Bootstrap } & \multicolumn{2}{|c|}{ Population } \\
\hline & Mean(slope) & $\mathrm{CI}^{95 \%}$ (slope) & $\operatorname{mean}(\mathrm{R} 2)$ in $\%$ & $\mathrm{CI}^{95 \%}(\mathrm{R} 2)$ & Slope & R2 (in\%) \\
\hline 2 & -0.07 & {$[-0.42,0.31]$} & 3.00 & {$[0.00,14.34]$} & -0.02 & 0.04 \\
\hline 3 & -0.07 & {$[-0.42,0.31]$} & 3.00 & {$[0.00,14.35]$} & -0.02 & 0.04 \\
\hline 4 & -0.07 & {$[-0.42,0.31]$} & 3.00 & {$[0.00,14.35]$} & -0.02 & 0.04 \\
\hline \multirow[t]{3}{*}{5} & -0.07 & {$[-0.42,0.31]$} & 3.00 & {$[0.00,14.35]$} & -0.02 & 0.04 \\
\hline & \multicolumn{6}{|c|}{ Calibration II } \\
\hline & \multicolumn{4}{|c|}{ Bootstrap } & \multicolumn{2}{|c|}{ Population } \\
\hline Horizon & Mean(slope) & $\mathrm{CI}^{95 \%}$ (slope) & $\operatorname{mean}(\mathrm{R} 2)$ in $\%$ & $\mathrm{CI}^{95 \%}(\mathrm{R} 2)$ & Slope & R2 (in\%) \\
\hline 2 & 0.05 & {$[-0.80,0.66]$} & 5.20 & {$[0.00,28.41]$} & 0.20 & 1.60 \\
\hline 3 & 0.06 & {$[-0.88,0.68]$} & 5.16 & {$[0.01,28.37]$} & 0.23 & 1.95 \\
\hline 4 & 0.06 & {$[-0.92,0.68]$} & 5.10 & {$[0.01,27.87]$} & 0.24 & 2.12 \\
\hline \multirow[t]{3}{*}{5} & 0.07 & {$[-0.94,0.69]$} & 5.08 & {$[0.00,27.72]$} & 0.25 & 2.21 \\
\hline & \multicolumn{6}{|c|}{ Calibration III } \\
\hline & \multicolumn{4}{|c|}{ Bootstrap } & \multicolumn{2}{|c|}{ Population } \\
\hline Horizon & Mean(slope) & $\mathrm{CI}^{95 \%}$ (slope) & mean $(\mathrm{R} 2)$ in $\%$ & $\mathrm{CI}^{95 \%}(\mathrm{R} 2)$ & Slope & R2 (in\%) \\
\hline 2 & -0.11 & {$[-0.90,0.53]$} & 4.46 & {$[0.00,22.52]$} & 0.03 & 0.02 \\
\hline 3 & -0.13 & {$[-1.09,0.56]$} & 4.67 & {$[0.00,24.82]$} & 0.05 & 0.07 \\
\hline 4 & -0.14 & {$[-1.19,0.58]$} & 4.81 & {$[0.01,25.87]$} & 0.06 & 0.12 \\
\hline \multirow[t]{3}{*}{5} & -0.14 & {$[-1.26,0.59]$} & 4.87 & {$[0.00,26.54]$} & 0.07 & 0.14 \\
\hline & \multicolumn{6}{|c|}{ Calibration III with $\gamma=5$} \\
\hline & \multicolumn{4}{|c|}{ Bootstrap } & \multicolumn{2}{|c|}{ Population } \\
\hline Horizon & Mean(slope) & $\mathrm{CI}^{95 \%}$ (slope) & $\operatorname{mean}(\mathrm{R} 2)$ in $\%$ & $\mathrm{CI}^{95 \%}(\mathrm{R} 2)$ & Slope & R2 (in\%) \\
\hline 2 & -0.39 & {$[-1.76,0.54]$} & 5.45 & {$[0.01,29.99]$} & -0.12 & 0.22 \\
\hline 3 & -0.44 & {$[-2.16,0.54]$} & 5.61 & {$[0.00,33.14]$} & -0.10 & 0.15 \\
\hline 4 & -0.46 & {$[-2.37,0.53]$} & 5.69 & {$[0.01,34.37]$} & -0.09 & 0.12 \\
\hline 5 & -0.47 & {$[-2.48,0.53]$} & 5.72 & {$[0.01,35.11]$} & -0.08 & 0.11 \\
\hline
\end{tabular}

NOTE - The bootstrap is based on 10,000 experiments, each running a regression

$$
h p r(t+1 ; t+h)-y(t ; 1)=a+b[f(t ; t+h)-y(t ; 1)]+\varepsilon(t+1 ; T)
$$

over 40 non-overlapping years with $h=2,3,4,5$. The population moments are based on 100,000 years of nonoverlapping data (the same regression). 
Is the negative sign of Fama-Bliss regressions (in calibration IV, for instance) for real bonds inconsistent with the results for nominal ones? Not necessarily. We know that the forward-spot spread is a (noisy) measure of expected returns. We also know from Fama and Bliss that it varies over the business cycles where positive forward-spot spreads tend to be common in booms. To the extent that the forward-spot spread predicts the future inflation risk premia in nominal bonds which have also business-cycle dynamics, we hypothesize that the negative sign for real bonds may be perfectly consistent with Fama and Bliss results for nominal bonds, as such a correlation may easily flip over the sign.

\section{Conclusion}

This paper develops a dynamic rational expectations general equilibrium model in order to explain a series of salient features of equity and fixed income markets. A particular focus is placed upon accounting for the overreaction and underreaction to macroeconomic news, volatility clustering, time-varying expected excess returns as well as matching the moments of the equity premium and the risk-free rate. The model features an unobservable dividend growth rate switching between two states at random times which leads to an interesting "signal extraction" problem. In addition, investors have a preference for early resolution of uncertainty, modeled by means of recursive homothetic Epstein-Zin preferences. The paper shows that the blend of these two features enriches the

canonical Lucas-tree economy sufficiently to succeed in accounting for the aforementioned stylized facts. 


\section{Appendix}

\section{A Steady-State Distribution of the Posterior Probability}

The Kolmogorov forward equation implies that the steady-state distribution $p(\pi)$ of the posterior probability is the unique solution of the second-order ordinary differential equation

$$
0=\frac{1}{2} \frac{d^{2}}{d \pi^{2}}\left[\left(\widetilde{\boldsymbol{\Sigma}}_{\pi} \widetilde{\boldsymbol{\Sigma}}_{\pi}^{\prime}\right) p(\pi)\right]-\frac{d}{d \pi}\left[(\lambda+\mu)\left(\pi^{s}-\pi\right) p(\pi)\right]
$$

subject to

$$
\int_{0}^{1} p(\pi) d \pi=1
$$

It may be shown that $\partial(0,1)$ are entrance boundaries; that is, they cannot be reached from the interior of the state space (Karatzas and Shreve (1991)). This implies that

$$
\begin{aligned}
& p(0)=0 \\
& p(1)=0
\end{aligned}
$$

Therefore, integrating the above equation from 0 to $\pi$ yields

$$
0=\frac{1}{2} \frac{d}{d \pi}\left[\left(\widetilde{\boldsymbol{\Sigma}}_{\pi} \widetilde{\boldsymbol{\Sigma}}_{\pi}^{\prime}\right) p(\pi)\right]-\left[(\lambda+\mu)\left(\pi^{s}-\pi\right) p(\pi)\right]
$$

We deal with the integral constraint by introducing the cumulative steady-state distribution function

$$
q(\pi)=\int_{0}^{\pi} p(s) d s
$$

Or, in differential form,

$$
\frac{d}{d \pi} q(\pi)=p(\pi)
$$

Clearly,

$$
\begin{aligned}
& q(0)=0 \\
& q(1)=1
\end{aligned}
$$

This allows us to recast the problem as a vector-valued linear boundary value problem in the unknown functions $(p(\pi), q(\pi))$

$$
\begin{aligned}
0 & =\frac{1}{2} \frac{d}{d \pi}\left[\left(\widetilde{\boldsymbol{\Sigma}}_{\pi} \widetilde{\boldsymbol{\Sigma}}_{\pi}^{\prime}\right) p(\pi)\right]-\left[(\lambda+\mu)\left(\pi^{s}-\pi\right) p(\pi)\right] \\
0 & =\frac{d}{d \pi} q(\pi)-p(\pi)
\end{aligned}
$$

We convert this system by differentiation to a homogeneous second-order ODE for the unknown cumulative distribution function $q(\pi)$

$$
0=\frac{1}{2}\left(\widetilde{\boldsymbol{\Sigma}}_{\pi} \widetilde{\boldsymbol{\Sigma}}_{\pi}^{\prime}\right) \frac{d^{2}}{d \pi^{2}} q(\pi)+\left[\left(\widetilde{\boldsymbol{\Sigma}}_{\pi} \widetilde{\boldsymbol{\Sigma}}_{\pi}^{\prime}\right) \frac{(1-2 \pi)}{\pi(1-\pi)}-(\lambda+\mu)\left(\pi_{s}-\pi\right)\right] \frac{d}{d \pi} q(\pi)
$$

subject to $q(0)=0, q(1)=1$. We solve this equation using finite difference methods on a regular mesh. Afterwards, we recover the steady-state density $p(\pi)$ as a numerical derivative of the approximate solution for the cumulative distribution function $q(\pi)$. 


\section{B Long-Run Uncertainty}

Let $\{\mathcal{I}(t)\}$ be the filteration generated by the past history of consumption, dividend, and additional signals $\left\{C_{s}, D_{s}, e_{s}\right\}_{s=0}^{t}$, which describes the information set of investors. The filteration $\{\mathcal{F}(t)\}$ describes full information of the economy, which contains additional information of the true state, $\left\{C_{s}, D_{s}, e_{s}, \theta_{s}\right\}_{s=0}^{t}$. We define the long-run consumption growth rate as

$$
G(T ; t)=\int_{t}^{T} \theta(s) d s
$$

Then, long-run uncertainty under investors' information set is

$$
V(t, T)=\operatorname{Var}[G(T ; t) \mid \mathcal{I}(t)]=\mathbb{E}_{t}^{\mathcal{I}}\left[G(T ; t)^{2}\right]-\mathbb{E}_{t}^{\mathcal{I}}[G(T ; t)]^{2} .
$$

We compute this value as follows.

First, the dynamics of the posterior probability is given by the diffusion

$$
d \pi=(\lambda+\mu)\left(\pi^{s}-\pi\right) d t+\widetilde{\mathbf{\Sigma}}_{\pi} d \widetilde{\mathbf{Z}}
$$

Integrating this equation from time $t$ to $T$ and taking conditional expectation yields

$$
\mathbb{E}_{t}^{\mathcal{I}}\left(\pi_{T}\right)=e^{-(\lambda+\mu)(T-t)} \pi_{t}+\left(1-e^{-(\lambda+\mu)(T-t)}\right) \pi^{s}
$$

Next, under standard regularity conditions,

$$
\begin{aligned}
\mathbb{E}_{t}^{\mathcal{I}}[G(T ; t)] & =\int_{t}^{T} \mathbb{E}_{t}^{\mathcal{I}}\left(\theta_{s}\right) d s=\int_{t}^{T} \mathbb{E}_{t}^{\mathcal{I}} \mathbb{E}_{s}^{\mathcal{I}}\left[\theta_{s}\right] d s \\
& =\int_{t}^{T} \mathbb{E}_{t}\left[\bar{\theta} \pi_{s}+\underline{\theta}\left(1-\pi_{s}\right)\right] d s \\
& =\underline{\theta}(T-t)+(\bar{\theta}-\underline{\theta}) \int_{t}^{T}\left\{\pi_{t} e^{-(\lambda+\mu)(s-t)}+\pi^{s}\left(1-e^{-(\lambda+\mu)(s-t)}\right)\right\} d s \\
& =\underline{\theta}(T-t)+(\bar{\theta}-\underline{\theta})(T-t) \pi^{s}+\left(\frac{\bar{\theta}-\underline{\theta}}{\lambda+\mu}\right)\left(\pi_{t}-\pi^{s}\right)\left(1-e^{-(\lambda+\mu)(T-t)}\right)
\end{aligned}
$$

To compute the second-moment of the long-run consumption growth rate, we first apply Ito lemma to $G(T ; t)$,

$$
d_{T} G^{2}(T ; t)=2 G(T ; t) d_{T} G(T ; t)=2 G(T ; t) \theta_{T} d T
$$

where the second equality follows from the definition of the long-run consumption growth rate. Upon integration from $t$ up to some capital $T$ (with obvious abuse of notation), we get that

$$
G^{2}(T ; t)-G^{2}(t ; t)=2 \int_{t}^{T} G(s ; t) \theta_{s} d s=2 \int_{t}^{T} d s \int_{t}^{s} d u \theta_{u} \theta_{s}
$$

Apply Fubini theorem,

$$
\mathbb{E}_{t}^{\mathcal{I}}\left[G^{2}(T ; t)\right]=2 \int_{t}^{T} d s \int_{t}^{s} d u \mathbb{E}_{t}^{\mathcal{I}}\left[\theta_{u} \theta_{s}\right]
$$

Note that $u<s$ and thus we can invoke the law of iterated expectations

$$
\mathbb{E}_{t}^{\mathcal{I}}\left[\theta_{u} \theta_{s}\right]=\mathbb{E}_{t}^{\mathcal{I}}\left[\mathbb{E}_{u}^{\mathcal{I}}\left[\theta_{u} \theta_{s}\right]\right]
$$

The inner expectation

$$
\mathbb{E}_{u}^{\mathcal{I}}\left[\theta_{u} \theta_{s}\right]=\mathbb{E}_{u}^{\mathcal{I}}\left[\mathbb{E}_{u}\left[\theta_{u} \theta_{s}\right]\right]=\mathbb{E}_{u}^{\mathcal{I}}\left[\theta_{u} \mathbb{E}_{u}\left[\theta_{s}\right]\right]
$$


where

$$
\mathbb{E}_{u}\left[\theta_{s}\right]=\left\{\begin{array}{ll}
\bar{\theta} P_{11}(s-u)+\underline{\theta} P_{12}(s-u), & \theta_{u}=\bar{\theta} \\
\bar{\theta} P_{21}(s-u)+\underline{\theta} P_{22}(s-u), & \theta_{u}=\underline{\theta}
\end{array},\right.
$$

and the transition probabilities $P_{i j}(s-u)$ satisfy

$$
[\mathbf{P}(s-u)]_{i j}=P_{i j}(s-u)=\left[e^{\Lambda(s-u)}\right]_{i j},
$$

where $\Lambda$ is the infinitesimal generator of Markov Chain for the expected consumption growth rate.

Substituting into equation (??) yields

$$
\mathbb{E}_{u}^{\mathcal{I}}\left[\theta_{u} \theta_{s}\right]=\bar{\theta}\left[\bar{\theta} P_{11}(s-u)+\underline{\theta} P_{12}(s-u)\right] \pi_{u}+\underline{\theta}\left[\bar{\theta} P_{21}(s-u)+\underline{\theta} P_{22}(s-u)\right]\left(1-\pi_{u}\right),
$$

which can be written in matrix form:

$$
\mathbb{E}_{u}^{\mathcal{I}}\left[\theta_{u} \theta_{s}\right]=\underline{\theta} \mathbf{P}_{2}(s-u)[\bar{\theta}, \underline{\theta}]^{\prime}+[\bar{\theta},-\underline{\theta}] \mathbf{P}(s-u)[\bar{\theta}, \underline{\theta}]^{\prime} \pi_{u}
$$

Computing the outer expectation in eq. (??) yields

$$
\begin{aligned}
\mathbb{E}_{t}^{\mathcal{I}}\left[\theta_{u} \theta_{s}\right] & =\underline{\theta} \iota_{2}^{\prime} \mathbf{P}(s-u)[\bar{\theta}, \underline{\theta}]^{\prime}+[\bar{\theta},-\underline{\theta}] \mathbf{P}(s-u)[\bar{\theta}, \underline{\theta}]^{\prime} \mathbb{E}\left[\pi_{u} \mid \mathcal{I}(t)\right] \\
& =\underline{\theta} \iota_{2}^{\prime} e^{\Lambda(s-u)}[\bar{\theta}, \underline{\theta}]^{\prime}+[\bar{\theta},-\underline{\theta}] e^{\mathbf{\Lambda}(s-u)}[\bar{\theta}, \underline{\theta}]^{\prime}\left[\pi_{t} e^{-(\lambda+\mu)(u-t)}+\pi^{s}\left(1-e^{-(\lambda+\mu)(u-t)}\right)\right]
\end{aligned}
$$

Finally, the double integral in (B.7) can be easily computed in closed form. The results are available upon request.

\section{Wealth-Consumption Ratio}

The wealth portfolio of the representative agent is the same as the claim on all the future consumption, where consumption can be viewed as dividend. We assume that its dynamics is given by:

$$
\frac{d W}{W}=\left[\mu_{W}(\pi)-\frac{C}{W}\right] d t+\sigma_{W, c}(\pi) d \widetilde{Z}^{c}+\sigma_{W, e}(\pi) d \widetilde{Z}^{e}
$$

In addition, we assume there is a riskless bond with interest rate $r(\pi)$ :

$$
d B=B r(\pi) d t
$$

Next, we solve the consumption-portfolio choice problem of the representative agent. Define the value function of the agent as:

$$
J(W, \pi)=\max _{\{C, x\}} \mathbb{E}\left[\int_{t}^{\infty} f\left(C_{s}, J_{s}\right) d s \mid \mathcal{I}(t)\right] .
$$

The agent chooses to hold $x$ shares of the consumptio claim, leaving the rest of the wealth in riskfree bonds. Her budget constraint is:

$$
d W=\left[r W+W x\left(\mu_{W}-r\right)-C\right] d t+W x\left(\sigma_{W, c} d \widetilde{Z}^{c}+\sigma_{W, e} d \widetilde{Z}^{e}\right)
$$


The Hamilton-Jacobi-Bellman equation is:

$$
\begin{aligned}
0= & f(C, J)+J_{W}\left[r W+W x\left(\mu_{W}-r\right)-C\right]+\frac{1}{2} J_{W W} W^{2} x^{2} \sigma_{W}^{2} \\
& +J_{\pi}(\lambda+\mu)\left(\pi^{s}-\pi\right)+\frac{1}{2} J_{\pi \pi} v^{2}(\pi)\left(\frac{1}{\sigma_{c}^{2}}+\frac{1}{\sigma_{e}^{2}}\right)+J_{W \pi} x W v(\pi)\left[\frac{\sigma_{W, c}}{\sigma_{c}}+\frac{\sigma_{W, e}}{\sigma_{e}}\right]
\end{aligned}
$$

where

$$
\sigma_{W}^{2}=\sigma_{W, c}^{2}+\sigma_{W, e}^{2}
$$

We guess the solution:

$$
J(W, \pi)=\delta^{\frac{\alpha}{\rho}} \Psi(\pi)^{\frac{\alpha}{\rho}(1-\rho)} \frac{W^{\alpha}}{\alpha},
$$

then

$$
\begin{aligned}
J_{W} & =\delta^{\frac{\alpha}{\rho}} \Psi(\pi)^{\frac{\alpha}{\rho}(1-\rho)} W^{\alpha-1}=\alpha J W^{-1} \\
J_{W W} & =\alpha(\alpha-1) J W^{-2} \\
J_{\pi} & =\delta^{\frac{\alpha}{\rho}} \frac{\alpha}{\rho}(1-\rho) \Psi(\pi)^{\frac{\alpha}{\rho}(1-\rho)-1} \Psi^{\prime} \frac{W^{\alpha}}{\alpha}=\frac{\alpha}{\rho}(1-\rho) \frac{\Psi^{\prime}}{\Psi} J \\
J_{\pi \pi} & =\delta^{\frac{\alpha}{\rho}} \frac{\alpha}{\rho}(1-\rho)\left[\Psi(\pi)^{\frac{\alpha}{\rho}(1-\rho)-1} \Psi^{\prime \prime}+\left(\frac{\alpha}{\rho}(1-\rho)-1\right) \Psi(\pi)^{\frac{\alpha}{\rho}(1-\rho)-2}\left(\Psi^{\prime}\right)^{2}\right] \frac{W^{\alpha}}{\alpha} \\
& =\frac{\alpha}{\rho}(1-\rho)\left[\frac{\Psi^{\prime \prime}}{\Psi}+\left(\frac{\alpha}{\rho}(1-\rho)-1\right)\left(\frac{\Psi^{\prime}}{\Psi}\right)^{2}\right] J \\
J_{W \pi} & =\frac{\alpha}{\rho}(1-\rho) \frac{\Psi^{\prime}}{\Psi} \alpha J W^{-1}
\end{aligned}
$$

From the HJB equation, the FOCs are:

$$
\begin{aligned}
f_{C} & =J_{W} \\
0 & =J_{W} W\left(\mu_{W}-r\right)+J_{W W} W^{2} x\left(\sigma_{W, c}^{2}+\sigma_{W, e}^{2}\right)+J_{W \pi} W v\left(\frac{\sigma_{W, c}}{\sigma_{c}}+\frac{\sigma_{W, e}}{\sigma_{e}}\right) .
\end{aligned}
$$

The second FOC implies:

$$
\begin{aligned}
x^{*}(\pi) & =-\frac{J_{W}}{W J_{W W}} \frac{\mu_{w}-r}{\sigma_{W}^{2}}-\frac{J_{W \pi} v}{J_{W W} W \sigma_{W}^{2}}\left(\frac{\sigma_{W, c}}{\sigma_{c}}+\frac{\sigma_{W, e}}{\sigma_{e}}\right) \\
& =\frac{1}{1-\alpha} \frac{\mu_{w}-r}{\sigma_{W}^{2}}-\frac{\frac{\alpha}{\rho}(1-\rho)}{(\alpha-1) \sigma_{W}^{2}}\left(\frac{\sigma_{W, c}}{\sigma_{c}}+\frac{\sigma_{W, e}}{\sigma_{e}}\right) v \frac{\Psi^{\prime}}{\Psi}
\end{aligned}
$$

This FOC also helps simplify the HJB:

$$
\begin{aligned}
0= & f(C, J)+J_{W}[r W-C]-\frac{1}{2} J_{W W} W^{2} x^{* 2} \sigma_{W}^{2} \\
& +J_{\pi}(\lambda+\mu)\left(\pi^{s}-\pi\right)+\frac{1}{2} J_{\pi \pi} v^{2}(\pi)\left(\frac{1}{\sigma_{c}^{2}}+\frac{1}{\sigma_{e}^{2}}\right) .
\end{aligned}
$$


From the consumption FOC:

$$
f_{C}=\delta \frac{C^{\rho-1}}{(\alpha J)^{\frac{\rho}{\alpha}-1}}=\alpha J W^{-1}
$$

or

$$
\begin{aligned}
C^{\rho-1} & =\delta^{-1}(\alpha J)^{\frac{\rho}{\alpha}} W^{-1} \\
& =\delta^{-1}\left(\delta^{\frac{\alpha}{\rho}} \Psi(\pi)^{\frac{\alpha}{\rho}(1-\rho)} W^{\alpha}\right)^{\frac{\rho}{\alpha}} W^{-1} \\
& =\delta^{-1} \delta \Psi(\pi)^{1-\rho} W^{\rho-1} \\
& =\Psi(\pi)^{1-\rho} W^{\rho-1}
\end{aligned}
$$

or

$$
W=\Psi(\pi) C
$$

Applying Ito's lemma,

$$
\begin{aligned}
d W & =\Psi d C+\Psi^{\prime} C d \pi+\Psi^{\prime \prime} C(d \pi)^{2}+\Psi^{\prime} d C d \pi \\
& =\Psi C \frac{d C}{C}+\Psi C \frac{\Psi^{\prime}}{\Psi} d \pi+\Psi C \frac{\Psi^{\prime \prime}}{\Psi}(d \pi)^{2}+\Psi C \frac{\Psi^{\prime}}{\Psi} \frac{d C}{C} d \pi
\end{aligned}
$$

or

$$
\begin{aligned}
\frac{d W}{W}= & \frac{d C}{C}+\frac{\Psi^{\prime}}{\Psi} d \pi+\frac{1}{2} \frac{\Psi^{\prime \prime}}{\Psi}(d \pi)^{2}+\frac{\Psi^{\prime}}{\Psi} \frac{d C}{C} d \pi \\
= & (\pi \bar{\theta}+(1-\pi) \underline{\theta}) d t+\sigma_{c} d \widetilde{Z}^{c} \\
& +\frac{\Psi^{\prime}}{\Psi}(\lambda+\mu)\left(\pi^{s}-\pi\right) d t+\frac{\Psi^{\prime}}{\Psi} v(\pi)\left[\frac{1}{\sigma_{c}} d \widetilde{Z}^{c}+\frac{1}{\sigma_{e}} d \widetilde{Z}^{e}\right] \\
& +\frac{1}{2} \frac{\Psi^{\prime \prime}}{\Psi} v^{2}(\pi)\left(\frac{1}{\sigma_{c}^{2}}+\frac{1}{\sigma_{e}^{2}}\right) d t+\frac{\Psi^{\prime}}{\Psi} v(\pi) d t \\
= & {\left[(\pi \bar{\theta}+(1-\pi) \underline{\theta})+\frac{\Psi^{\prime}}{\Psi}(\lambda+\mu)\left(\pi^{s}-\pi\right)+\frac{1}{2} \frac{\Psi^{\prime \prime}}{\Psi} v^{2}(\pi)\left(\frac{1}{\sigma_{c}^{2}}+\frac{1}{\sigma_{e}^{2}}\right)+\frac{\Psi^{\prime}}{\Psi} v(\pi)\right] d t } \\
& +\left(\sigma_{c}+\frac{\Psi^{\prime}}{\Psi} \frac{v(\pi)}{\sigma_{c}}\right) d \widetilde{Z}^{c}+\frac{\Psi^{\prime}}{\Psi} \frac{v(\pi)}{\sigma_{e}} d \widetilde{Z}^{e} .
\end{aligned}
$$

Thus,

$$
\begin{aligned}
\sigma_{W, c}(\pi) & =\sigma_{c}+\frac{\Psi^{\prime}}{\Psi} \frac{v(\pi)}{\sigma_{c}}, \\
\sigma_{W, e}(\pi) & =\frac{\Psi^{\prime}}{\Psi} \frac{v(\pi)}{\sigma_{e}}
\end{aligned}
$$

and

$$
\mu_{W}(\pi)-\Psi^{-1}(\pi)=(\pi \bar{\theta}+(1-\pi) \underline{\theta})+\frac{\Psi^{\prime}}{\Psi}(\lambda+\mu)\left(\pi^{s}-\pi\right)+\frac{1}{2} \frac{\Psi^{\prime \prime}}{\Psi} v^{2}(\pi)\left(\frac{1}{\sigma_{c}^{2}}+\frac{1}{\sigma_{e}^{2}}\right)+\frac{\Psi^{\prime}}{\Psi} v(\pi) .
$$


Also, we can rewrite the aggregator as:

$$
\begin{aligned}
f(C, J) & =\frac{\delta}{\rho}\left(\frac{C^{\rho}}{(\alpha J)^{\frac{\rho}{\alpha}-1}}-\alpha J\right)=\frac{\delta}{\rho}\left(\delta^{-1} \frac{C}{W}-1\right) \alpha J \\
& =\frac{\delta}{\rho}\left(\delta^{-1} \Psi(\pi)^{-1}-1\right) \alpha J
\end{aligned}
$$

So, plug $f$ and the derivatives of $J$ into the HJB equation,

$$
\begin{aligned}
0= & \frac{\delta}{\rho}\left(\delta^{-1} \Psi(\pi)^{-1}-1\right) \alpha J+\alpha J W^{-1}[r W-C]-\frac{1}{2} \alpha(\alpha-1) J W^{-2} W^{2} x^{* 2} \sigma_{W}^{2} \\
& +\frac{\alpha}{\rho}(1-\rho) \frac{\Psi^{\prime}}{\Psi} J(\lambda+\mu)\left(\pi^{s}-\pi\right) \\
& +\frac{1}{2} \frac{\alpha}{\rho}(1-\rho)\left[\frac{\Psi^{\prime \prime}}{\Psi}+\left(\frac{\alpha}{\rho}(1-\rho)-1\right)\left(\frac{\Psi^{\prime}}{\Psi}\right)^{2}\right] J v^{2}\left(\frac{1}{\sigma_{c}^{2}}+\frac{1}{\sigma_{e}^{2}}\right)
\end{aligned}
$$

or

$$
\begin{aligned}
0= & \left(\frac{1}{\rho} \Psi(\pi)^{-1}-\frac{\delta}{\rho}\right) \alpha+\alpha\left[r-\Psi(\pi)^{-1}\right]-\frac{1}{2} \alpha(\alpha-1) x^{* 2} \sigma_{W}^{2} \\
& +\frac{\alpha}{\rho}(1-\rho) \frac{\Psi^{\prime}}{\Psi}(\lambda+\mu)\left(\pi^{s}-\pi\right) \\
& +\frac{1}{2} \frac{\alpha}{\rho}(1-\rho)\left[\frac{\Psi^{\prime \prime}}{\Psi}+\left(\frac{\alpha}{\rho}(1-\rho)-1\right)\left(\frac{\Psi^{\prime}}{\Psi}\right)^{2}\right] v^{2}\left(\frac{1}{\sigma_{c}^{2}}+\frac{1}{\sigma_{e}^{2}}\right)
\end{aligned}
$$

Next, we impose the market clearing conditions on the consumption:

$$
x=1,
$$

which implies:

$$
\begin{aligned}
1= & \frac{1}{1-\alpha} \frac{\mu_{w}-r}{\sigma_{W}^{2}}-\frac{\frac{\alpha}{\rho}(1-\rho)}{(\alpha-1) \sigma_{W}^{2}}\left(\frac{\sigma_{W, c}}{\sigma_{c}}+\frac{\sigma_{W, e}}{\sigma_{e}}\right) v \frac{\Psi^{\prime}}{\Psi} \\
& \frac{1}{1-\alpha} \frac{\mu_{W}-r}{\sigma_{W}^{2}}-\frac{\frac{\alpha}{\rho}(1-\rho)}{(\alpha-1) \sigma_{W}} \frac{\Psi^{\prime}}{\Psi} v(\pi)
\end{aligned}
$$


or

$$
\begin{aligned}
\mu_{W}(\pi)-r(\pi)= & (1-\alpha) \sigma_{W}^{2}(\pi)-\frac{\alpha}{\rho}(1-\rho) \frac{\Psi^{\prime}}{\Psi}\left(\frac{\sigma_{W, c}(\pi)}{\sigma_{c}}+\frac{\sigma_{W, e}(\pi)}{\sigma_{e}}\right) v(\pi) \\
= & (1-\alpha)\left(\sigma_{W, c}^{2}+\sigma_{W, e}^{2}\right)-\frac{\alpha}{\rho}(1-\rho) \frac{\Psi^{\prime}}{\Psi}\left(\frac{\sigma_{W, c}}{\sigma_{c}}+\frac{\sigma_{W, e}}{\sigma_{e}}\right) v(\pi) \\
= & {\left[(1-\alpha) \sigma_{W, c}-\frac{\alpha}{\rho}(1-\rho) \frac{\Psi^{\prime}}{\Psi} \frac{v(\pi)}{\sigma_{c}}\right] \sigma_{W, c} } \\
& +\left[(1-\alpha) \sigma_{W, e}-\frac{\alpha}{\rho}(1-\rho) \frac{\Psi^{\prime}}{\Psi} \frac{v(\pi)}{\sigma_{e}}\right] \sigma_{W, e} \\
= & {\left[(1-\alpha)\left(\sigma_{c}+\frac{\Psi^{\prime}}{\Psi} \frac{v(\pi)}{\sigma_{c}}\right)-\frac{\alpha}{\rho}(1-\rho) \frac{\Psi^{\prime}}{\Psi} \frac{v(\pi)}{\sigma_{c}}\right] \sigma_{W, c} } \\
& +\left[(1-\alpha) \frac{\Psi^{\prime}}{\Psi} \frac{v(\pi)}{\sigma_{e}}-\frac{\alpha}{\rho}(1-\rho) \frac{\Psi^{\prime}}{\Psi} \frac{v(\pi)}{\sigma_{e}}\right] \sigma_{W, e}, \\
= & {\left[(1-\alpha) \sigma_{c}+\left(1-\frac{\alpha}{\rho}\right) \frac{\Psi^{\prime}}{\Psi} \frac{v(\pi)}{\sigma_{c}}\right] \sigma_{W, c}+\left(1-\frac{\alpha}{\rho}\right) \frac{\Psi^{\prime}}{\Psi} \frac{v(\pi)}{\sigma_{e}} \sigma_{W, e} . }
\end{aligned}
$$

which simplifies to:

$$
\mu_{W}(\pi)-r(\pi)=\left[(1-\alpha) \sigma_{c}+\left(1-\frac{\alpha}{\rho}\right) \frac{\Psi^{\prime}}{\Psi} \frac{v(\pi)}{\sigma_{c}}\right] \sigma_{W, c}+\left(1-\frac{\alpha}{\rho}\right) \frac{\Psi^{\prime}}{\Psi} \frac{v(\pi)}{\sigma_{e}} \sigma_{W, e}
$$

Here, both the consumption shocks and the shocks for the additional signal are priced. The risk price for the consumption shock has two components: the first part is the standard risk price for local consumption shocks; the second part is the uncertainty price. Similarly, the risk price for the shocks on the signal is also related to investor uncertainty. Moreover, the equilibrium interest rate is:

$$
\begin{aligned}
r(\pi)= & \mu_{W}(\pi)-\left[(1-\alpha) \sigma_{c}+\left(1-\frac{\alpha}{\rho}\right) \frac{\Psi^{\prime}}{\Psi} \frac{v(\pi)}{\sigma_{c}}\right] \sigma_{W, c}-\left(1-\frac{\alpha}{\rho}\right) \frac{\Psi^{\prime}}{\Psi} \frac{v(\pi)}{\sigma_{e}} \sigma_{W, e} \\
= & \Psi^{-1}(\pi)+(\pi \bar{\theta}+(1-\pi) \underline{\theta})+\frac{\Psi^{\prime}}{\Psi}(\lambda+\mu)\left(\pi^{s}-\pi\right)+\frac{1}{2} \frac{\Psi^{\prime \prime}}{\Psi} v^{2}(\pi)\left(\frac{1}{\sigma_{c}^{2}}+\frac{1}{\sigma_{e}^{2}}\right)+\frac{\Psi^{\prime}}{\Psi} v(\pi) \\
& -\left[(1-\alpha) \sigma_{c}+\left(1-\frac{\alpha}{\rho}\right) \frac{\Psi^{\prime}}{\Psi} \frac{v(\pi)}{\sigma_{c}}\right] \sigma_{W, c}-\left(1-\frac{\alpha}{\rho}\right) \frac{\Psi^{\prime}}{\Psi} \frac{v(\pi)}{\sigma_{e}} \sigma_{W, e} \\
r(\pi)= & \mu_{W}(\pi)-(1-\alpha) \sigma_{W}^{2}(\pi)+\frac{\alpha}{\rho}(1-\rho) \frac{\Psi^{\prime}}{\Psi}\left(\frac{\sigma_{W, c}(\pi)}{\sigma_{c}}+\frac{\sigma_{W, e}(\pi)}{\left.\sigma_{e}\right) v(\pi)}\right. \\
= & \Psi^{-1}(\pi)+(\pi \bar{\theta}+(1-\pi) \underline{\theta})+\frac{\Psi^{\prime}}{\Psi}(\lambda+\mu)\left(\pi^{s}-\pi\right)+\frac{1}{2} \frac{\Psi^{\prime \prime}}{\Psi} v^{2}(\pi)\left(\frac{1}{\sigma_{c}^{2}}+\frac{1}{\sigma_{e}^{2}}\right)+\frac{\Psi^{\prime}}{\Psi} v(\pi) \\
& -(1-\alpha) \sigma_{W}^{2}(\pi)+\frac{\alpha}{\rho}(1-\rho) \frac{\Psi^{\prime}}{\Psi}\left(\frac{\sigma_{W, c}(\pi)}{\sigma_{c}}+\frac{\sigma_{W, e}(\pi)}{\sigma_{e}}\right) v(\pi)
\end{aligned}
$$


Finally, we can substitute the equilibrium conditions back into the HJB equation:

$$
\begin{aligned}
0= & \left(\frac{1}{\rho} \Psi(\pi)^{-1}-\frac{\delta}{\rho}\right) \alpha+\alpha\left[r-\Psi(\pi)^{-1}\right]-\frac{1}{2} \alpha(\alpha-1) \sigma_{W}^{2} \\
& +\frac{\alpha}{\rho}(1-\rho) \frac{\Psi^{\prime}}{\Psi}(\lambda+\mu)\left(\pi^{s}-\pi\right) \\
& +\frac{1}{2} \frac{\alpha}{\rho}(1-\rho)\left[\frac{\Psi^{\prime \prime}}{\Psi}+\left(\frac{\alpha}{\rho}(1-\rho)-1\right)\left(\frac{\Psi^{\prime}}{\Psi}\right)^{2}\right] v^{2}\left(\frac{1}{\sigma_{c}^{2}}+\frac{1}{\sigma_{e}^{2}}\right)
\end{aligned}
$$

or

$$
\begin{aligned}
0= & \left(\frac{1}{\rho} \Psi(\pi)^{-1}-\frac{\delta}{\rho}\right) \alpha \\
& +\alpha\left[\begin{array}{c}
\left.(\pi \bar{\theta}+(1-\pi) \underline{\theta})+\frac{\Psi^{\prime}}{\Psi}(\lambda+\mu)\left(\pi^{s}-\pi\right)+\frac{1}{2} \frac{\Psi^{\prime \prime}}{\Psi} v^{2}(\pi)\left(\frac{1}{\sigma_{c}^{2}}+\frac{1}{\sigma_{e}^{2}}\right)+\frac{\Psi^{\prime}}{\Psi} v(\pi)\right] \\
-(1-\alpha) \sigma_{W}^{2}(\pi)+\frac{\alpha}{\rho}(1-\rho) \frac{\Psi^{\prime}}{\Psi}\left(\frac{\sigma_{W, c}(\pi)}{\sigma_{c}}+\frac{\sigma_{W, e}(\pi)}{\sigma_{e}}\right) v(\pi)
\end{array}\right] \\
& -\frac{1}{2} \alpha(\alpha-1) \sigma_{W}^{2}+\frac{\alpha}{\rho}(1-\rho) \frac{\Psi^{\prime}}{\Psi}(\lambda+\mu)\left(\pi^{s}-\pi\right) \\
& +\frac{1}{2} \frac{\alpha}{\rho}(1-\rho)\left[\frac{\Psi^{\prime \prime}}{\Psi}+\left(\frac{\alpha}{\rho}(1-\rho)-1\right)\left(\frac{\Psi^{\prime}}{\Psi}\right)^{2}\right] v^{2}\left(\frac{1}{\sigma_{c}^{2}}+\frac{1}{\sigma_{e}^{2}}\right)
\end{aligned}
$$

or

$$
\begin{aligned}
0= & \left(\frac{1}{\rho} \Psi(\pi)^{-1}-\frac{\delta}{\rho}\right) \\
& +\left[\begin{array}{c}
\left.(\pi \bar{\theta}+(1-\pi) \underline{\theta})+\frac{\Psi^{\prime}}{\Psi}(\lambda+\mu)\left(\pi^{s}-\pi\right)+\frac{1}{2} \frac{\Psi^{\prime \prime}}{\Psi} v^{2}(\pi)\left(\frac{1}{\sigma_{c}^{2}}+\frac{1}{\sigma_{e}^{2}}\right)+\frac{\Psi^{\prime}}{\Psi} v(\pi)\right] \\
+\frac{\alpha}{\rho}(1-\rho) \frac{\Psi^{\prime}}{\Psi}\left(\frac{\sigma_{W, c}(\pi)}{\sigma_{c}}+\frac{\sigma_{W, e}(\pi)}{\sigma_{e}}\right) v(\pi)
\end{array}\right] \\
& +\frac{1}{2}(\alpha-1) \sigma_{W}^{2}+\frac{1-\rho}{\rho} \frac{\Psi^{\prime}}{\Psi}(\lambda+\mu)\left(\pi^{s}-\pi\right) \\
& +\frac{1}{2} \frac{1-\rho}{\rho}\left[\frac{\Psi^{\prime \prime}}{\Psi}+\left(\frac{\alpha}{\rho}(1-\rho)-1\right)\left(\frac{\Psi^{\prime}}{\Psi}\right)^{2}\right] v^{2}\left(\frac{1}{\sigma_{c}^{2}}+\frac{1}{\sigma_{e}^{2}}\right)
\end{aligned}
$$

or

$$
\begin{aligned}
0= & \left(\frac{1}{\rho} \Psi(\pi)^{-1}-\frac{\delta}{\rho}\right) \\
& +\left[\begin{array}{c}
\left.(\pi \bar{\theta}+(1-\pi) \underline{\theta})+\frac{\Psi^{\prime}}{\Psi}(\lambda+\mu)\left(\pi^{s}-\pi\right)+\frac{1}{2} \frac{\Psi^{\prime \prime}}{\Psi} v^{2}(\pi)\left(\frac{1}{\sigma_{c}^{2}}+\frac{1}{\sigma_{e}^{2}}\right)+\frac{\Psi^{\prime}}{\Psi} v(\pi)\right] \\
+\frac{\alpha}{\rho}(1-\rho) \frac{\Psi^{\prime}}{\Psi}\left(1+\frac{\Psi^{\prime}}{\Psi} \frac{v(\pi)}{\sigma_{c}^{2}}+\frac{\Psi^{\prime}}{\Psi} \frac{v(\pi)}{\sigma_{e}^{2}}\right) v(\pi)
\end{array}\right] \\
& +\frac{1}{2}(\alpha-1)\left[\left(\sigma_{c}+\frac{\Psi^{\prime}}{\Psi} \frac{v(\pi)}{\sigma_{c}}\right)^{2}+\left(\frac{\Psi^{\prime}}{\Psi} \frac{v(\pi)}{\sigma_{e}}\right)^{2}\right]+\frac{1-\rho}{\rho} \frac{\Psi^{\prime}}{\Psi}(\lambda+\mu)\left(\pi^{s}-\pi\right) \\
& +\frac{1}{2} \frac{1-\rho}{\rho}\left[\frac{\Psi^{\prime \prime}}{\Psi}+\left(\frac{\alpha}{\rho}(1-\rho)-1\right)\left(\frac{\Psi^{\prime}}{\Psi}\right)^{2}\right] v^{2}\left(\frac{1}{\sigma_{c}^{2}}+\frac{1}{\sigma_{e}^{2}}\right)
\end{aligned}
$$


or

$$
\begin{aligned}
0= & \frac{1}{\rho}-\frac{\delta}{\rho} \Psi(\pi) \\
& +(\pi \bar{\theta}+(1-\pi) \underline{\theta}) \Psi(\pi) \\
& +\Psi^{\prime}(\lambda+\mu)\left(\pi^{s}-\pi\right)+\frac{1}{2} \Psi^{\prime \prime} v^{2}(\pi)\left(\frac{1}{\sigma_{c}^{2}}+\frac{1}{\sigma_{e}^{2}}\right)+v(\pi) \Psi^{\prime} \\
& +\frac{\alpha}{\rho}(1-\rho)\left(1+\frac{\Psi^{\prime}}{\Psi} \frac{v(\pi)}{\sigma_{c}^{2}}+\frac{\Psi^{\prime}}{\Psi} \frac{v(\pi)}{\sigma_{e}^{2}}\right) v(\pi) \Psi^{\prime} \\
& +\frac{1}{2}(\alpha-1)\left(\sigma_{c}+\frac{\Psi^{\prime}}{\Psi} \frac{v(\pi)}{\sigma_{c}}\right)^{2} \Psi(\pi)+\frac{1}{2}(\alpha-1)\left(\frac{\Psi^{\prime}}{\Psi} \frac{v(\pi)}{\sigma_{e}}\right)^{2} \Psi(\pi) \\
& +\frac{1-\rho}{\rho} \Psi^{\prime}(\lambda+\mu)\left(\pi^{s}-\pi\right) \\
& +\frac{1}{2} \frac{1-\rho}{\rho}\left[\Psi^{\prime \prime}+\left(\frac{\alpha}{\rho}(1-\rho)-1\right) \frac{\left(\Psi^{\prime}\right)^{2}}{\Psi}\right] v^{2}\left(\frac{1}{\sigma_{c}^{2}}+\frac{1}{\sigma_{e}^{2}}\right)
\end{aligned}
$$

or

$$
\begin{aligned}
0= & \frac{1}{\rho} \\
+ & {\left[-\frac{\delta}{\rho}+(\pi \bar{\theta}+(1-\pi) \underline{\theta})+\frac{1}{2}(\alpha-1) \sigma_{c}^{2}\right] \Psi } \\
+ & {\left[(\lambda+\mu)\left(\pi^{s}-\pi\right)+v(\pi)+\frac{\alpha}{\rho}(1-\rho) v(\pi)+(\alpha-1) v(\pi)+\frac{1-\rho}{\rho}(\lambda+\mu)\left(\pi^{s}-\pi\right)\right] \Psi^{\prime} } \\
+ & {\left[\frac{\alpha}{\rho}(1-\rho)+\frac{1}{2} \frac{1-\rho}{\rho}\left(\frac{\alpha}{\rho}(1-\rho)-1\right)+\frac{1}{2}(\alpha-1)\right]\left(\frac{1}{\sigma_{c}^{2}}+\frac{1}{\sigma_{e}^{2}}\right) v^{2}(\pi) \frac{\left(\Psi^{\prime}\right)^{2}}{\Psi} } \\
+ & {\left[\frac{1-\rho}{\rho}+1\right] v^{2}(\pi)\left(\frac{1}{\sigma_{c}^{2}}+\frac{1}{\sigma_{e}^{2}}\right) \frac{1}{2} \Psi^{\prime \prime} } \\
0=\frac{1}{\rho} & +\left[-\frac{\delta}{\rho}+(\pi \bar{\theta}+(1-\pi) \underline{\theta})+\frac{1}{2}(\alpha-1) \sigma_{c}^{2}\right] \Psi \\
& +\left[\frac{\alpha}{\rho} v(\pi)+\frac{1}{\rho}(\lambda+\mu)\left(\pi^{s}-\pi\right)\right] \Psi^{\prime} \\
& +\frac{1}{2} \frac{\alpha-\rho}{\rho^{2}}\left(\frac{1}{\sigma_{c}^{2}}+\frac{1}{\sigma_{e}^{2}}\right) v^{2}(\pi) \frac{\left(\Psi^{\prime}\right)^{2}}{\Psi}+\frac{1}{\rho} v^{2}(\pi)\left(\frac{1}{\sigma_{c}^{2}}+\frac{1}{\sigma_{e}^{2}}\right) \frac{1}{2} \Psi^{\prime \prime}
\end{aligned}
$$

or

$$
\begin{aligned}
0= & 1+\left[-\delta+\rho(\pi \bar{\theta}+(1-\pi) \underline{\theta})+\frac{1}{2} \rho(\alpha-1) \sigma_{c}^{2}\right] \Psi \\
& +\left[\alpha v(\pi)+(\lambda+\mu)\left(\pi^{s}-\pi\right)\right] \Psi^{\prime} \\
& +\frac{1}{2}\left(\frac{\alpha}{\rho}-1\right)\left(\frac{1}{\sigma_{c}^{2}}+\frac{1}{\sigma_{e}^{2}}\right) v^{2}(\pi) \frac{\left(\Psi^{\prime}\right)^{2}}{\Psi}+\frac{1}{2}\left(\frac{1}{\sigma_{c}^{2}}+\frac{1}{\sigma_{e}^{2}}\right) v^{2}(\pi) \Psi^{\prime \prime}
\end{aligned}
$$


with boundary conditions:

$$
\begin{aligned}
& 0=1+\left[-\delta+\rho(\mu+\underline{\theta})+\frac{1}{2} \rho(\alpha-1) \sigma_{c}^{2}\right] \Psi(0)+\mu \Psi^{\prime}(0) \\
& 0=1+\left[-\delta+\rho(\mu+\bar{\theta})+\frac{1}{2} \rho(\alpha-1) \sigma^{2}\right] \Psi(1)-\lambda \Psi^{\prime}(1)
\end{aligned}
$$

Next, for the real riskfree rate,

$$
\begin{gathered}
\sigma_{W, c}(\pi)=\sigma_{c}+\frac{\Psi^{\prime}}{\Psi} \frac{v(\pi)}{\sigma_{c}}, \\
\sigma_{W, e}(\pi)=\frac{\Psi^{\prime}}{\Psi} \frac{v(\pi)}{\sigma_{e}}, \\
r(\pi)=\Psi^{-1}(\pi)+(\pi \bar{\theta}+(1-\pi) \underline{\theta})+\frac{\Psi^{\prime}}{\Psi}(\lambda+\mu)\left(\pi^{s}-\pi\right)+\frac{1}{2} \frac{\Psi^{\prime \prime}}{\Psi} v^{2}(\pi)\left(\frac{1}{\sigma_{c}^{2}}+\frac{1}{\sigma_{e}^{2}}\right)+\frac{\Psi^{\prime}}{\Psi} v(\pi) \\
-(1-\alpha) \sigma_{W}^{2}(\pi)+\frac{\alpha}{\rho}(1-\rho) \frac{\Psi^{\prime}}{\Psi}\left(\frac{\sigma_{W, c}(\pi)}{\sigma_{c}}+\frac{\sigma_{W, e}(\pi)}{\sigma_{e}}\right) v(\pi) \\
=\Psi^{-1}(\pi)+(\pi \bar{\theta}+(1-\pi) \underline{\theta})+\frac{\Psi^{\prime}}{\Psi}(\lambda+\mu)\left(\pi^{s}-\pi\right)+\frac{1}{2} \frac{\Psi^{\prime \prime}}{\Psi} v^{2}(\pi)\left(\frac{1}{\sigma_{c}^{2}}+\frac{1}{\sigma_{e}^{2}}\right)+\frac{\Psi^{\prime}}{\Psi} v(\pi)(\mathrm{C} .23) \\
-\left[(1-\alpha) \sigma_{c}+\left(1-\frac{\alpha}{\rho}\right) \frac{\Psi^{\prime}}{\Psi} \frac{v(\pi)}{\sigma_{c}}\right]\left(\sigma_{c}+\frac{\Psi^{\prime}}{\Psi} \frac{v(\pi)}{\sigma_{c}}\right)-\left(1-\frac{\alpha}{\rho}\right) \frac{\Psi^{\prime}}{\Psi} \frac{v(\pi)}{\sigma_{e}} \frac{\Psi^{\prime}}{\Psi} \frac{v(\pi)}{\sigma_{e}}
\end{gathered}
$$

From the ODE:

$$
\begin{aligned}
\Psi(\pi)^{-1}= & -\left[-\delta+\rho(\pi \bar{\theta}+(1-\pi) \underline{\theta})+\frac{1}{2} \rho(\alpha-1) \sigma_{c}^{2}\right]-\left[\alpha v(\pi)+(\lambda+\mu)\left(\pi^{s}-\pi\right)\right] \frac{\Psi^{\prime}}{\Psi} \\
& -\frac{1}{2}\left(\frac{\alpha}{\rho}-1\right)\left(\frac{1}{\sigma_{c}^{2}}+\frac{1}{\sigma_{e}^{2}}\right) v^{2}(\pi)\left(\frac{\Psi^{\prime}}{\Psi}\right)^{2}-\frac{1}{2}\left(\frac{1}{\sigma_{c}^{2}}+\frac{1}{\sigma_{e}^{2}}\right) v^{2}(\pi) \frac{\Psi^{\prime \prime}}{\Psi}
\end{aligned}
$$


Then,

$$
\begin{aligned}
& r(\pi)=-\left[-\delta+\rho(\pi \bar{\theta}+(1-\pi) \underline{\theta})+\frac{1}{2} \rho(\alpha-1) \sigma_{c}^{2}\right]-\left[\alpha v(\pi)+(\lambda+\mu)\left(\pi^{s}-\pi\right)\right] \frac{\Psi^{\prime}}{\Psi} \\
& -\frac{1}{2}\left(\frac{\alpha}{\rho}-1\right)\left(\frac{1}{\sigma_{c}^{2}}+\frac{1}{\sigma_{e}^{2}}\right) v^{2}(\pi)\left(\frac{\Psi^{\prime}}{\Psi}\right)^{2}-\frac{1}{2}\left(\frac{1}{\sigma_{c}^{2}}+\frac{1}{\sigma_{e}^{2}}\right) v^{2}(\pi) \frac{\Psi^{\prime \prime}}{\Psi} \\
& +(\pi \bar{\theta}+(1-\pi) \underline{\theta})+\frac{\Psi^{\prime}}{\Psi}(\lambda+\mu)\left(\pi^{s}-\pi\right)+\frac{1}{2} \frac{\Psi^{\prime \prime}}{\Psi} v^{2}(\pi)\left(\frac{1}{\sigma_{c}^{2}}+\frac{1}{\sigma_{e}^{2}}\right)+\frac{\Psi^{\prime}}{\Psi} v(\pi) \\
& -\left[(1-\alpha) \sigma_{c}+\left(1-\frac{\alpha}{\rho}\right) \frac{\Psi^{\prime}}{\Psi} \frac{v(\pi)}{\sigma_{c}}\right]\left(\sigma_{c}+\frac{\Psi^{\prime}}{\Psi} \frac{v(\pi)}{\sigma_{c}}\right)-\left(1-\frac{\alpha}{\rho}\right) \frac{\Psi^{\prime}}{\Psi} \frac{v(\pi)}{\sigma_{e}} \frac{\Psi^{\prime}}{\Psi} \frac{v(\pi)}{\sigma_{e}} \\
& =\delta+(1-\rho)(\pi \bar{\theta}+(1-\pi) \underline{\theta})-\frac{1}{2} \rho(\alpha-1) \sigma_{c}^{2}-(1-\alpha) \sigma_{c}^{2} \\
& -\left[\alpha v(\pi)+(\lambda+\mu)\left(\pi^{s}-\pi\right)\right] \frac{\Psi^{\prime}}{\Psi}+\frac{\Psi^{\prime}}{\Psi}(\lambda+\mu)\left(\pi^{s}-\pi\right) \\
& +\frac{\Psi^{\prime}}{\Psi} v(\pi)-\left(1-\frac{\alpha}{\rho}\right) \frac{\Psi^{\prime}}{\Psi} v(\pi)-(1-\alpha) \frac{\Psi^{\prime}}{\Psi} v(\pi) \\
& +\frac{1}{2} \frac{\Psi^{\prime \prime}}{\Psi} v^{2}(\pi)\left(\frac{1}{\sigma_{c}^{2}}+\frac{1}{\sigma_{e}^{2}}\right)-\frac{1}{2}\left(\frac{1}{\sigma_{c}^{2}}+\frac{1}{\sigma_{e}^{2}}\right) v^{2}(\pi) \frac{\Psi^{\prime \prime}}{\Psi} \\
& -\frac{1}{2}\left(\frac{\alpha}{\rho}-1\right)\left(\frac{1}{\sigma_{c}^{2}}+\frac{1}{\sigma_{e}^{2}}\right) v^{2}(\pi)\left(\frac{\Psi^{\prime}}{\Psi}\right)^{2} \\
& -\left(1-\frac{\alpha}{\rho}\right)\left(\frac{\Psi^{\prime}}{\Psi}\right)^{2} \frac{v^{2}(\pi)}{\sigma_{c}^{2}}-\left(1-\frac{\alpha}{\rho}\right)\left(\frac{\Psi^{\prime}}{\Psi}\right)^{2} \frac{v^{2}(\pi)}{\sigma_{e}^{2}} \\
& =\delta+(1-\rho)(\pi \bar{\theta}+(1-\pi) \underline{\theta})-\frac{1}{2}(1-\alpha)(2-\rho) \sigma_{c}^{2} \\
& -\left(1-\frac{\alpha}{\rho}\right) \frac{\Psi^{\prime}}{\Psi} v(\pi)-\frac{1}{2}\left(1-\frac{\alpha}{\rho}\right)\left(\frac{\Psi^{\prime}}{\Psi}\right)^{2}\left(\frac{1}{\sigma_{c}^{2}}+\frac{1}{\sigma_{e}^{2}}\right) v^{2}(\pi)
\end{aligned}
$$

which simplifies to:

$$
\begin{aligned}
r(\pi)= & \delta+(1-\rho)(\pi \bar{\theta}+(1-\pi) \underline{\theta})-\frac{1}{2}(1-\alpha)(2-\rho) \sigma_{c}^{2} \\
& -\left(1-\frac{\alpha}{\rho}\right) \frac{\Psi^{\prime}}{\Psi} v(\pi)-\frac{1}{2}\left(1-\frac{\alpha}{\rho}\right)\left(\frac{1}{\sigma_{c}^{2}}+\frac{1}{\sigma_{e}^{2}}\right) v^{2}(\pi)\left(\frac{\Psi^{\prime}}{\Psi}\right)^{2}
\end{aligned}
$$

\section{Levered Stock}

For the leveraged stock,

$$
\frac{d D}{D}=\left[\theta_{m}+\phi\left(\widehat{\theta}-\theta_{m}\right)\right] d t+\phi \sigma_{c} d \widetilde{Z}^{c}+\sigma_{d} d Z^{d}
$$

We guess:

$$
S(D, \pi)=D \Pi(\pi)
$$

Then, $S$ satisfies:

$$
\frac{d S}{S}=\left[\mu_{S}(\pi)-\Pi^{-1}(\pi)\right] d t+\sigma_{S, c}(\pi) d \widetilde{Z}^{c}+\sigma_{S, e}(\pi) d \widetilde{Z}^{e}+\sigma_{S, d} d Z^{d} .
$$

Consumption Euler equation implies:

$$
\mu_{S}(\pi)-r(\pi)=\eta_{c}(\pi) \sigma_{S, c}(\pi)+\eta_{e}(\pi) \sigma_{S, e}(\pi) .
$$


Apply Ito's lemma to $S(D, \pi)$,

$$
\begin{aligned}
& d S=\Pi d D+D \Pi^{\prime} d \pi+\frac{1}{2} D \Pi^{\prime \prime} d \pi^{2}+\Pi^{\prime} d D d \pi \\
& d \pi=(\lambda+\mu)\left(\pi^{s}-\pi\right) d t+v(\pi)\left[\frac{1}{\sigma_{c}} d \widetilde{Z}^{c}+\frac{1}{\sigma_{e}} d \widetilde{Z}^{e}\right], \\
& \frac{d S}{S}=\frac{d D}{D}+\frac{\Pi^{\prime}}{\Pi} d \pi+\frac{1}{2} \frac{\Pi^{\prime \prime}}{\Pi} d \pi^{2}+\frac{\Pi^{\prime}}{\Pi} \frac{d D}{D} d \pi \\
&=\quad\left[\theta_{m}+\phi\left(\widehat{\theta}-\theta_{m}\right)\right] d t+\phi \sigma_{c} d \widetilde{Z}^{c}+\sigma_{d} d Z^{d} \\
&+\frac{\Pi^{\prime}}{\Pi}(\lambda+\mu)\left(\pi^{s}-\pi\right) d t+\frac{\Pi^{\prime}}{\Pi} v(\pi)\left[\frac{1}{\sigma_{c}} d \widetilde{Z}^{c}+\frac{1}{\sigma_{e}} d \widetilde{Z}^{e}\right] \\
&+\frac{1}{2} \frac{\Pi^{\prime \prime}}{\Pi} v^{2}(\pi)\left(\frac{1}{\sigma_{c}^{2}}+\frac{1}{\sigma_{e}^{2}}\right) d t+\frac{\Pi^{\prime}}{\Pi} \phi v(\pi) d t \\
& \quad\left[\theta_{m}+\phi\left(\widehat{\theta}-\theta_{m}\right)+\frac{\Pi^{\prime}}{\Pi}(\lambda+\mu)\left(\pi^{s}-\pi\right)+\frac{1}{2} \frac{\Pi^{\prime \prime}}{\Pi} v^{2}(\pi)\left(\frac{1}{\sigma_{c}^{2}}+\frac{1}{\sigma_{e}^{2}}\right)+\frac{\Pi^{\prime}}{\Pi} \phi v(\pi)\right] d t \\
&+\left(\phi \sigma_{c}+\frac{\Pi^{\prime}}{\Pi} \frac{v(\pi)}{\sigma_{c}}\right) d \widetilde{Z}^{c}+\frac{\Pi^{\prime}}{\Pi} \frac{v(\pi)}{\sigma_{e}} d \widetilde{Z}^{e}+\sigma_{d} d Z^{d}
\end{aligned}
$$

Thus, we have an ODE for $\Pi(\pi)$ over the domain $[0,1]$ :

$$
\begin{aligned}
& \theta_{m}+\phi\left(\widehat{\theta}-\theta_{m}\right)+\frac{\Pi^{\prime}}{\Pi}(\lambda+\mu)\left(\pi^{s}-\pi\right)+\frac{1}{2} \frac{\Pi^{\prime \prime}}{\Pi} v^{2}(\pi)\left(\frac{1}{\sigma_{c}^{2}}+\frac{1}{\sigma_{e}^{2}}\right)+\frac{\Pi^{\prime}}{\Pi} \phi v(\pi)+\frac{1}{\Pi}-r(\pi) \\
= & \eta_{c}(\pi)\left(\phi \sigma_{c}+\frac{\Pi^{\prime}}{\Pi} \frac{v(\pi)}{\sigma_{c}}\right)+\eta_{e}(\pi) \frac{\Pi^{\prime}}{\Pi} \frac{v(\pi)}{\sigma_{e}}
\end{aligned}
$$

or

$$
\begin{aligned}
& {\left[(1-\phi) \theta_{m}+\phi \hat{\theta}\right] \Pi+(\lambda+\mu)\left(\pi^{s}-\pi\right) \Pi^{\prime}+\frac{1}{2} v^{2}(\pi)\left(\frac{1}{\sigma_{c}^{2}}+\frac{1}{\sigma_{e}^{2}}\right) \Pi^{\prime \prime}+\phi v(\pi) \Pi^{\prime}+1-r(\pi) \Pi } \\
= & \eta_{c}(\pi) \phi \sigma_{c} \Pi+\eta_{c}(\pi) \frac{v(\pi)}{\sigma_{c}} \Pi^{\prime}+\eta_{e}(\pi) \frac{v(\pi)}{\sigma_{e}} \Pi^{\prime}
\end{aligned}
$$

or

$$
\begin{aligned}
0= & 1+\left[(1-\phi) \theta_{m}+\phi(\pi \bar{\theta}+(1-\pi) \underline{\theta})-r(\pi)-\eta_{c}(\pi) \phi \sigma_{c}\right] \Pi \\
& +\left[(\lambda+\mu)\left(\pi^{s}-\pi\right)+\phi v(\pi)-\left(\frac{\eta_{c}(\pi)}{\sigma_{c}}+\frac{\eta_{e}(\pi)}{\sigma_{e}}\right) v(\pi)\right] \Pi^{\prime} \\
& +\frac{1}{2} v^{2}(\pi)\left(\frac{1}{\sigma_{c}^{2}}+\frac{1}{\sigma_{e}^{2}}\right) \Pi^{\prime \prime}
\end{aligned}
$$

with boundary conditions:

$$
\begin{aligned}
\lim _{\pi \rightarrow \partial(0,1)} \Pi^{\prime} \pi(1-\pi) & =0 \\
\lim _{\pi \rightarrow \partial(0,1)} \Pi^{\prime \prime} \pi^{2}(1-\pi)^{2} & =0
\end{aligned}
$$

Notice that $r(\pi), \eta_{c}(\pi)$ and $\eta_{e}(\pi)$ are solved from the HJB equation earlier. Then, the boundary conditions can be written as:

$$
\begin{aligned}
& 0=1+\left[(1-\phi) \theta_{m}+\phi \underline{\theta}-r(0)-\eta_{c}(0) \phi \sigma_{c}\right] \Pi(0)+\mu \Pi^{\prime}(0) \\
& 0=1+\left[(1-\phi) \theta_{m}+\phi \bar{\theta}-r(1)-\eta_{c}(1) \phi \sigma_{c}\right] \Pi(1)-\lambda \Pi^{\prime}(1)
\end{aligned}
$$




\section{E Indexed Bond Pricing}

Because the preferences are homothetic and the problem is homogeneous, the bond price is only a function of the posterior probability $\pi(t)$ and time $t$, but not of the wealth $W(t)$, that is, $B(t ; T)=B(\pi(t), t ; T)$. The necessary condition for the interior optimum with respect to the share $a_{B}(t)$ of the wealth held in the indexed bond with maturity $T$ is

$$
\alpha_{B}(\pi, t ; T)-r(\pi)=-\frac{W \partial_{W W} J}{\partial_{W} J} g_{S}(\pi) g_{B}(\pi, t ; T)-\frac{\partial_{W \pi} J}{\partial_{W} J} g_{B}(\pi, t ; T) v(\pi)
$$

Recall the dynamics of the bond price

$$
\frac{d B(t ; T)}{B(t ; T)}=\alpha_{B}(t ; T) d t+g_{B}(t ; T) d \widetilde{Z}(t)
$$

Apply Itô lemma to $B(\pi, t ; T)$ and compare the drift and the volatility with the above equation, using the first-order condition (E.1), to get

$$
\begin{aligned}
\alpha_{B}(\pi, t ; T) & =[\lambda+\mu]\left[\pi^{s}-\pi\right] \frac{\partial_{\pi} B(\pi, t ; T)}{B(\pi, t ; T)}+\frac{1}{2} v^{2}(\pi) \frac{\partial_{\pi \pi} B(\pi, t ; T)}{B(\pi, t ; T)} \\
g_{B}(\pi, t ; T) & =v(\pi) \frac{\partial_{\pi} B(\pi, t ; T)}{B(\pi, t ; T)}
\end{aligned}
$$

and subsequently obtain the result that the zero-coupon bond price $B \in \mathcal{C}_{(0,1) \times(0, T)}^{2}$ satisfies the following backward parabolic partial differential equation

$$
0=\partial_{t} B+\beta_{2}(\pi) \partial_{\pi \pi} B+\beta_{1}(\pi) \partial_{\pi} B+\beta_{0}(\pi) B
$$

where

$$
\begin{aligned}
& \beta_{2}(\pi)=\frac{1}{2} v^{2}(\pi) \\
& \beta_{1}(\pi)=[\lambda+\mu]\left[\pi^{s}-\pi\right]-[1-\alpha] \sigma_{D} v(\pi)-[1-\alpha / \rho] v^{2}(\pi) \frac{\Psi^{\prime}(\pi)}{\Psi(\pi)} \\
& \beta_{0}(\pi)=-r(\pi)
\end{aligned}
$$

where $\Psi(\pi)$ is the equilibrium price-dividend ratio on the Lucas tree, and the real interest rate $r(\pi)$ is given by To determine the boundary conditions, we need to take a close look at the pricing equation as $\pi \downarrow 0$ and $\pi \uparrow 1$. We know that the value of the bond must be finite. We can use this condition to deduce boundary conditions from the PDE. First, we claim that

$$
\lim _{\pi \rightarrow \partial(0,1)} \beta_{2}(\pi) \partial_{\pi \pi} B=0
$$

Suppose not so that $\lim _{\pi \rightarrow \partial(0,1)} \beta_{2}(\pi) \partial_{\pi \pi} B=\mathcal{O}(1)$. For $\pi$ close to $\partial(0,1)$, we would have

$$
\partial_{\pi \pi} B=\mathcal{O}\left(\frac{1}{\pi^{2}(1-\pi)^{2}}\right)
$$

which upon integration shows that the bond price $B$ explodes as $\pi \rightarrow \partial(0,1)$.

Second, we claim that

$$
\lim _{\pi \rightarrow \partial(0,1)} \pi[1-\pi] \partial_{\pi} B=0
$$


Again, suppose not, then close to $\partial(0,1)$

$$
\partial_{\pi} B=\mathcal{O}\left(\frac{1}{\pi(1-\pi)}\right)
$$

and hence upon integration, the bond price explodes on the boundary. Similarly, one may show that

$$
\lim _{\pi \rightarrow \partial(0,1)} \pi^{2}[1-\pi]^{2} \partial_{\pi} B=0
$$

As a result, we conclude that the correct boundary conditions are

$$
\begin{aligned}
& 0=\partial_{t} B+[\lambda+\mu] \pi^{s} \partial_{\pi} B+\beta_{0}(0) B \\
& 0=\partial_{t} B+[\lambda+\mu]\left[\pi^{s}-1\right] \partial_{\pi} B+\beta_{0}(1) B
\end{aligned}
$$

In the special case of expected utility (i.e. $\alpha=\rho$ ), there exists an analytical solution. In fact, make the educated guess that

$$
B(\pi, t ; T)=[1-\alpha][\bar{\theta}-\underline{\theta}] \pi \Phi_{1}(t ; T)+\Phi_{2}(t ; T)
$$

Substitute this guess into the PDE and obtain the system of first-order ODEs and

$$
\begin{aligned}
& \underline{r}=\delta+[1-\alpha] \underline{\theta}-\frac{1}{2}(1-\alpha)[2-\alpha] \sigma_{D}^{2} \\
& \bar{r}=\underline{r}+[1-\alpha][\bar{\theta}-\underline{\theta}]
\end{aligned}
$$

The final condition for $\Phi_{1}(T ; T)$ and $\Phi_{2}(T ; T)$ dictated by $B(\pi, T ; T)=1$ is $\Phi_{1}(T ; T)=0$ and $\Phi_{2}(T ; T)=1$. The ODE system has a solution and upon substitution in the original guess we get the desired result.

\section{F Numerical Analysis of the Bond Pricing Equation: Crank-Nicholson Scheme}

The zero-coupon bond price function $B \in \mathcal{C}_{(0,1) \times(0, T)}^{2}$ satisfies the following backward parabolic PDE

$$
0=\partial_{t} B+\beta_{2}(\pi) \partial_{\pi \pi} B+\beta_{1}(\pi) \partial_{\pi} B+\beta_{0}(\pi) B
$$

subject to the final condition $B(\pi, T)=1$ and two boundary conditions at $\partial(0,1)$ of the form

$$
\begin{aligned}
& 0=\partial_{t} B+[\lambda+\mu] \pi^{s} \partial_{\pi} B+\beta_{0}(0) B \\
& 0=\partial_{t} B+[\lambda+\mu]\left[\pi^{s}-1\right] \partial_{\pi} B+\beta_{0}(1) B
\end{aligned}
$$

We approximate the partial derivatives using finite differences and solve the problem numerically using the Crank-Nicholson scheme. That is, we divide the $\pi$-axis into equally spaced nodes a distance $\delta \pi$ apart, and the $t$-axis into equally spaced nodes a distance $\delta t$ apart. This divides the $(0,1) \times(0, T)$ rectangle into a mesh, where the mesh points are of the form $(n \delta \pi, m \delta t), n=0, \ldots, N ; m=0, \ldots, M$ and $N=1 / \delta \pi, M=T / \delta T$. We write

$$
B_{n}^{m}=B(n \delta \pi, m \delta t)
$$

We use finite differences to obtain a discrete set of equations. The space derivatives are

$$
\begin{aligned}
\partial_{\pi} B(n \delta \pi, m \delta t) & =\frac{B_{n+1}^{m}-B_{n-1}^{m}}{2 \delta \pi}+\mathcal{O}\left([\delta \pi]^{2}\right) \\
\partial_{\pi \pi} B(n \delta \pi, m \delta t) & =\frac{B_{n+1}^{m}-2 B_{n}^{m}+B_{n-1}^{m}}{(\delta \pi)^{2}}+\mathcal{O}\left([\delta \pi]^{2}\right)
\end{aligned}
$$


Because the Crank-Nicholson scheme is essentially an average of the implicit and the explicit finite difference scheme, we approximate the time derivative using both backward and forward differences as

$$
\begin{aligned}
& \partial_{t} B(n \delta \pi, m \delta t)=\frac{B_{n}^{m+1}-B_{n}^{m}}{\delta t}+\mathcal{O}(\delta t) \\
& \partial_{t} B(n \delta \pi, m \delta t)=\frac{B_{n}^{m}-B_{n}^{m-1}}{\delta t}+\mathcal{O}(\delta t)
\end{aligned}
$$

The explicit finite-difference scheme for $n=1, \ldots, N-1$ is

$$
0=\frac{B_{n}^{m}-B_{n}^{m-1}}{\delta t}+\beta_{2}(\pi) \frac{B_{n+1}^{m}-2 B_{n}^{m}+B_{n-1}^{m}}{(\delta x)^{2}}+\beta_{1}(\pi) \frac{B_{n+1}^{m}-B_{n-1}^{m}}{2 \delta \pi}+\beta_{0}(\pi) B_{n}^{m}+\mathcal{O}\left(\delta t+[\delta \pi]^{2}\right)
$$

and the implicit finite-difference scheme for $n=1, \ldots, N-1$ is

$0=\frac{B_{n}^{m}-B_{n}^{m-1}}{\delta t}+\beta_{2}(\pi) \frac{B_{n+1}^{m-1}-2 B_{n}^{m-1}+B_{n-1}^{m-1}}{(\delta x)^{2}}+\beta_{1}(\pi) \frac{B_{n+1}^{m-1}-B_{n-1}^{m-1}}{2 \delta \pi}+\beta_{0}(\pi) B_{n}^{m-1}+\mathcal{O}\left(\delta t+[\delta \pi]^{2}\right)$

The famous Crank-Nicholson scheme is essentially a simple average of the explicit and implicit finite-difference schemes. It can be shown that it is precise up to $\mathcal{O}\left([\delta t]^{2}+[\delta \pi]^{2}\right)$. In order not to compromise the precision as well as the stability of the scheme, it is necessary to approximate the boundary conditions up to $\mathcal{O}\left([\delta t]^{2}+[\delta \pi]^{2}\right)$ as well. Thus, we make the following one-sided approximations

$$
\begin{gathered}
\partial_{\pi} B(0, m \delta t)=\frac{-B_{2}^{m}+4 B_{1}^{m}-3 B_{0}^{m}}{2 \delta \pi}+\mathcal{O}\left((\delta \pi)^{2}\right) \\
\partial_{\pi} B(1, m \delta t)=\frac{B_{N-2}^{m}-4 B_{N-1}^{m}+3 B_{N}^{m}}{2 \delta \pi}+\mathcal{O}\left((\delta \pi)^{2}\right)
\end{gathered}
$$

which is simple to prove using Taylor-series expansion. The code is implemented C.

\section{G Bayesian Analysis of the Two-State Univariate Regime-Switching Model for the Log-Consumption Growth Rate}

This appendix details the estimation of the Bayesian univariate regime-switching model, as applied to the growth rate of real per-capita nondurable consumption and services, denoted $C$. Let us recall the dynamics of the consumption growth rate

$$
\frac{d C(t)}{C(t)}=\theta(t) d t+\sigma_{C} d Z_{1}(t)
$$

where $Z_{1}(t)$ is a standard Wiener process. We discretize the above equation as follows

$$
\frac{\Delta C_{t}}{C_{t}}=\theta\left(s_{t}\right)+\sigma_{C} E_{t}
$$


where $E_{t} \sim$ i.i.d. : $N(0,1), s_{t}$ follows a two-state hidden Markov chain, and $\theta\left(s_{t}\right) \in\{\bar{\theta}, \underline{\theta}\}$, where $\bar{\theta}>\underline{\theta}$. Furthermore, let

$$
\begin{aligned}
\mathbf{Y} & =\left[\Delta \log C_{1947: Q 2}, \ldots, \Delta \log C_{2007: Q 2}\right]_{T \times 1}^{\prime} \\
\mathbf{s}_{1} & =\left[1\left(s_{1947: Q 2}=1\right), \ldots, 1\left(s_{2007: Q 2}=1\right)\right]_{T \times 1}^{\prime} \\
\mathbf{s}_{2} & =\left[1\left(s_{1947: Q 2}=2\right), \ldots, 1\left(s_{2007: Q 2}=2\right)\right]_{T \times 1}^{\prime} \\
\mathbf{X} & =\left[\mathbf{s}_{1}, \mathbf{s}_{2},\right]_{T \times 2}
\end{aligned}
$$

where $1(\bullet)$ is an indicator function for the simulated state $s_{t}$ at time $t$. In addition, let the matrix $B_{2 \times 1}$ of regression coefficients be given by

$$
\mathbf{B}=[\bar{\theta}, \underline{\theta}]_{2 \times 1}^{\prime}
$$

Stacking the data into matrices, the discretized model may be written as

$$
\mathbf{Y}=\mathbf{X} \mathbf{B}+\sigma_{C} \mathbf{E}
$$

\section{G.1 Generating $B$ and $\Sigma$ Conditional on the Rest of the Parameters and $s_{T}$}

Under the assumptions made above, the likelihood function $l\left(B, \sigma_{C}^{2} \mid Y, X\right)$ is

$$
\left.l\left(\mathbf{B}, \sigma_{C}^{2}\right) \mid \mathbf{y}, \mathbf{x}\right) \propto\left(\sigma_{C}^{2}\right)^{-T / 2} \times \exp \left\{-\frac{1}{2 \sigma_{C}^{2}}(\mathbf{Y}-\mathbf{X B})^{\prime}(\mathbf{Y}-\mathbf{X B})\right\}
$$

Noting that

$$
(\mathbf{Y}-\mathbf{X B})^{\prime}(\mathbf{Y}-\mathbf{X} \mathbf{B})=\mathbf{S}+(\mathbf{B}-\hat{\mathbf{B}})^{\prime} \mathbf{X}^{\prime} \mathbf{X}(\mathbf{B}-\hat{\mathbf{B}})
$$

where $\hat{B}$ is the OLS estimate

$$
\hat{\mathbf{B}}=\left(\mathbf{X}^{\prime} \mathbf{X}\right)^{-1} \mathbf{X}^{\prime} \mathbf{Y}
$$

and

$$
S=(\mathbf{Y}-\mathbf{X} \hat{\mathbf{B}})^{\prime}(\mathbf{Y}-\mathbf{X} \hat{\mathbf{B}})
$$

we find that the likelihood function takes the form

$$
l\left(\mathbf{B}, \sigma_{C}^{2} \mid \mathbf{Y}, \mathbf{X}\right) \propto\left(\sigma_{C}^{2}\right)^{-T / 2} \times \exp \left\{-\frac{1}{2 \sigma_{C}^{2}} S-\frac{1}{2 \sigma_{C}^{2}}(\mathbf{B}-\hat{\mathbf{B}})^{\prime} \mathbf{X}^{\prime} \mathbf{X}(\mathbf{B}-\hat{\mathbf{B}})\right\}
$$

The prior distribution of the consumption variance is assumed to be inverse Gamma $\mathrm{I} \Gamma\left(\nu_{0} / 2, \delta_{0} / 2\right)$, with the density

$$
p\left(\frac{1}{\sigma_{C}^{2}}\right) \propto\left(\frac{1}{\sigma_{C}^{2}}\right)^{\frac{\nu_{0}}{2}-1} \exp \left\{-\frac{\delta_{0}}{2 \sigma_{C}^{2}}\right\}
$$

and the prior for the consumption growth rates $B$ is multivariate Normal $N(\overline{\mathbf{B}}, C)$, having the density

$$
p(\mathbf{B}) \propto \exp \left\{-\frac{1}{2}(\mathbf{B}-\overline{\mathbf{B}})^{\prime} \mathbf{C}^{-1}(\mathbf{B}-\overline{\mathbf{B}})\right\}
$$

In addition, we assume that the priors for $B$ and $1 / \sigma_{C}^{2}$ are statistically independent, that is,

$$
\begin{aligned}
p\left(\frac{1}{\sigma_{C}^{2}}, \mathbf{B}\right) & =p\left(\frac{1}{\sigma_{C}^{2}}\right) \times p(\mathbf{B}) \\
& \propto\left(\frac{1}{\sigma_{C}^{2}}\right)^{\frac{\nu_{0}}{2}-1} \exp \left\{-\frac{\delta_{0}}{2 \sigma_{C}^{2}}-\frac{1}{2}(\mathbf{B}-\overline{\mathbf{B}})^{\prime} \mathbf{C}^{-1}(\mathbf{B}-\overline{\mathbf{B}})\right\}
\end{aligned}
$$


The joint posterior distribution is then as follows

$$
\begin{aligned}
p\left(\mathbf{B}, \frac{1}{\sigma_{C}^{2}} \mid \mathbf{Y}, \mathbf{X}\right) & \propto\left(\frac{1}{\sigma_{C}^{2}}\right)^{\frac{\nu_{0}+T}{2}-1} \exp \left\{-\frac{\delta_{0}}{2 \sigma_{C}^{2}}-\frac{1}{2}(\mathbf{B}-\overline{\mathbf{B}})^{\prime} \mathbf{C}^{-1}(\mathbf{B}-\overline{\mathbf{B}})\right\} \times \\
& \times \exp \left\{-\frac{1}{2 \sigma_{C}^{2}} S-\frac{1}{2 \sigma_{C}^{2}}(\mathbf{B}-\hat{\mathbf{B}})^{\prime} \mathbf{X}^{\prime} \mathbf{X}(\mathbf{B}-\hat{\mathbf{B}})\right\}
\end{aligned}
$$

Merging the two quadratic forms in the exponent yields

$$
p\left(\mathbf{B}, \frac{1}{\sigma_{C}^{2}} \mid \mathbf{Y}, \mathbf{X}\right) \propto\left(\frac{1}{\sigma_{C}^{2}}\right)^{\frac{\nu_{0}+T}{2}-1} \times \exp \left\{-\frac{\delta_{0}+S}{2 \sigma_{C}^{2}}-\frac{1}{2}(\mathbf{B}-\mathbf{d})^{\prime} \mathbf{D}^{-1}(\mathbf{B}-\mathbf{d})\right\}
$$

where

$$
\begin{aligned}
& \mathbf{D}=\left(\frac{1}{\sigma_{C}^{2}} \mathbf{X}^{\prime} \mathbf{X}+\mathbf{C}^{-1}\right)^{-1} \\
& \mathbf{d}=\mathbf{D} \times\left(\frac{1}{\sigma_{C}^{2}} \mathbf{X}^{\prime} \mathbf{X} \hat{\mathbf{B}}+\mathbf{C}^{-1} \overline{\mathbf{B}}\right)=\mathbf{D} \times\left(\frac{1}{\sigma_{C}^{2}} \mathbf{X}^{\prime} \mathbf{Y}+\mathbf{C}^{-1} \overline{\mathbf{B}}\right)
\end{aligned}
$$

Note that we can factor out the joint posterior as

$$
p\left(\mathbf{B}, \frac{1}{\sigma_{C}^{2}}, \mid \mathbf{Y}, \mathbf{X}\right)=p\left(\mathbf{B} \mid \frac{1}{\sigma_{C}^{2}}, \mathbf{Y}, \mathbf{X}\right) \times p\left(\frac{1}{\sigma_{C}^{2}} \mid \mathbf{Y}, \mathbf{X}\right),
$$

so that the conditional posterior $p\left(\mathbf{B} \mid \frac{1}{\sigma_{C}^{2}}, \mathbf{Y}, \mathbf{X}\right)$, which in fact we do need for the Gibbs sampler, is

$$
p\left(\mathbf{B} \mid \frac{1}{\sigma_{C}^{2}}, \mathbf{Y}, \mathbf{X}\right) \propto \exp \left\{-\frac{1}{2}(\mathbf{B}-\mathbf{d})^{\prime} \mathbf{D}^{-1}(\mathbf{B}-\mathbf{d})\right\}
$$

which we recognize as the density of the multivariate Normal distribution

$$
\mathbf{B} \mid \frac{1}{\sigma_{C}^{2}}, \mathbf{Y}, \mathbf{X} \sim N(\mathbf{d}, \mathbf{D})
$$

With respect to the conditional posterior of the inverse of the consumption growth rate variance $\sigma_{C}^{2}$, it is helpful to recall eq. (??) and rewrite the joint posterior in eq. G.15) as

$$
\begin{aligned}
p\left(\mathbf{B}, \frac{1}{\sigma_{C}^{2}} \mid \mathbf{Y}, \mathbf{X}\right) & \propto\left(\frac{1}{\sigma_{C}^{2}}\right)^{\frac{\nu_{0}+T}{2}-1} \exp \left\{-\frac{\delta_{0}}{2 \sigma_{C}^{2}}-\frac{1}{2}(\mathbf{B}-\overline{\mathbf{B}})^{\prime} \mathbf{C}^{-1}(\mathbf{B}-\overline{\mathbf{B}})\right\} \times \\
& \times \exp \left\{-\frac{1}{2 \sigma_{C}^{2}}(\mathbf{Y}-\mathbf{X B})^{\prime}(\mathbf{Y}-\mathbf{X B})\right\}
\end{aligned}
$$

or

$$
\begin{aligned}
p\left(\mathbf{B}, \frac{1}{\sigma_{C}^{2}} \mid \mathbf{Y}, \mathbf{X}\right) & \propto\left(\frac{1}{\sigma_{C}^{2}}\right)^{\frac{\nu_{0}+T}{2}-1} \exp \left\{-\frac{1}{2 \sigma_{C}^{2}}\left[\delta_{0}+(\mathbf{Y}-\mathbf{X B})^{\prime}(\mathbf{Y}-\mathbf{X B})\right]\right\} \times \\
& \times \exp \left\{-\frac{1}{2}(\mathbf{B}-\overline{\mathbf{B}})^{\prime} \mathbf{C}^{-1}(\mathbf{B}-\overline{\mathbf{B}})\right\}
\end{aligned}
$$

Note that we may factorize the joint posterior for $\left(\mathbf{B}, \frac{1}{\sigma_{C}^{2}}\right)$ also as

$$
p\left(\mathbf{B}, \frac{1}{\sigma_{C}^{2}} \mid \mathbf{Y}, \mathbf{X}\right)=p\left(\frac{1}{\sigma_{C}^{2}} \mid \mathbf{B}, \mathbf{Y}, \mathbf{X}\right) \times p(\mathbf{B} \mid \mathbf{Y}, \mathbf{X})
$$


This gives us the conditional distribution for $1 / \sigma_{C}^{2}$ as

$$
p\left(\frac{1}{\sigma_{C}^{2}} \mid \mathbf{B}, \mathbf{Y}, \mathbf{X}\right) \propto\left(\frac{1}{\sigma_{C}^{2}}\right)^{\frac{\nu_{0}+T}{2}-1} \exp \left\{-\frac{1}{2 \sigma_{C}^{2}}\left[\delta_{0}+(\mathbf{Y}-\mathbf{X B})^{\prime}(\mathbf{Y}-\mathbf{X B})\right]\right\}
$$

which we recognize as the density of the inverse Gamma distribution

$$
\frac{1}{\sigma_{C}^{2}} \mid \mathbf{B}, \mathbf{Y}, \mathbf{X} \sim I \Gamma\left[\frac{\nu_{0}+T}{2}, \frac{\delta_{0}+(\mathbf{Y}-\mathbf{X B})^{\prime}(\mathbf{Y}-\mathbf{X B})}{2}\right]
$$

\section{G.2 Generating Transition Probabilities Conditional on $s_{T}$}

\section{G.2.1 Finding the Posterior Distribution}

Observe that conditional on $s_{T}$, the transition probabilities $\left(\pi_{i, j}\right)_{2 x 2}$ are independent of the data set $Y$ and the model's other parameters. We assume that the rows of the transition probability matrix are a priori independent, each having the Dirichlet distribution

$$
\begin{aligned}
\left(\pi_{i, 1}, \pi_{i, 2}\right) & \sim \operatorname{Dir}\left(\delta_{i, 1}, \delta_{i, 2} ; 2\right),:::: i=1,2 \\
& \propto \pi_{i, 1}^{\delta_{i, 1}} \times\left(1-\pi_{i, 1}\right)^{\delta_{i, 2}}
\end{aligned}
$$

The likelihood function is

$$
l\left(\left\{\pi_{i j}\right\}_{i, j=1}^{2} \mid \mathbf{s}_{T}\right)=\prod_{i, j=1}^{2} \pi_{i, j}^{n_{i, j}}
$$

where $n_{i, j}$ is the total number of transitions from state $i$ to state $j$. Hence, the posterior distribution takes the form

$$
\left(\pi_{i, 1}, \pi_{i, 2} \mid \mathbf{s}_{T}\right) \sim \operatorname{Dir}\left(\delta_{i, 1}+n_{i, 1}, \delta_{i, 2},+n_{i, 2} ; 2\right),::: i=1,2
$$

Clearly, the rows of the transition matrix are conditionally independent.

Sampling from the Dirichlet distribution $\operatorname{Dir}\left(\delta_{1}, \delta_{2} ; 2\right)$ is relatively simple. One first generates independent $\xi_{1}, \xi_{2}$ from the Gamma distribution $\Gamma\left(\delta_{i}, 1\right)$. Then, the couple

$$
\left(\frac{\xi_{i}}{\sum_{j=1}^{2} \xi_{j}}\right)_{i=1,2}
$$

has the desired Dirichlet distribution.

\section{G.2.2 Testing the Embeddability Conditions of the Sampled Transition Probability Matrix}

Luckily, the sufficient conditions for the existence of an infinitesimal generator that corresponds to a given sampled $2 \times 2$ transition probability matrix have been identified. They are

$$
\begin{aligned}
\operatorname{det}(P) & >0 \\
\operatorname{trace}(P) & >1
\end{aligned}
$$

We have found their imposition to be informative. 


\section{References}

Abel, A. B., 1999, "Risk premia and term premia in general equilibrium," Journal of Monetary Economics, 43, 3-33.

Ai, H., 2007, "Information Quality and Long-run Risk: Asset Pricing and Welfare Implications," Working Paper, Duke University.

Bansal, R., R. F. Dittmar, and C. Lundblad, 2005, "Consumption, Dividends, and the Cross-Section of Equity Returns," Journal of Finance, 60, 1639-1672.

Bansal, R., and I. Shaliastovich, 2008, "Learning, Long-Run Risks and Asset Price Jumps," Working Paper, Duke University.

Bansal, R., and A. Yaron, 2004, "Risks for the Long Run: A Potential Resolution of Asset Pricing Puzzles," Journal of Finance, 59, 1481 - 1509.

Bollerslev, T., and H. Zhou, 2007, "Expected stock returns and variance risk premia," Working Paper.

Brennan, M. J., and Y. Xia, 2001, "Stock Price Volatility and the Equity Premium," Journal of Monetary Economics, 47, 249-283.

Cagetti, M., L. P. Hansen, T. J. Sargent, and N. Williams, 2002, "Robustness and pricing with uncertain growth," The Review of Financial Studies, 15, 363-404.

Chen, H., 2007, "Macroeconomic Conditions and the Puzzles of Credit Spreads and Capital Structure," Working Paper, Massachusetts Institute of Technology.

David, A., 1997, "Fluctuating confidence in stock markets: Implications for returns and volatility," Journal of Financial and Quantitative Analysis, 32, 427-462.

Detemple, J., 1986, "Asset pricing in a production economy with incomplete information," Journal of Finance, 41, 383-391.

Dothan, M. U., and D. Feldman, 1986, "Equilibrium interest rates and multiperiod bonds in a partially observable economy," Journal of Finance, 41, 369-382.

Drechsler, I., and A. Yaron, 2007, "What's Vol Got to Do With It," Working Paper, Wharton School.

Duffie, D., and L. G. Epstein, 1992, "Stochastic Differential Utility," Econometrica, 60, 353-394.

Duffie, D., M. Schroder, and C. Skiadas, 1997, "A Term Structure Model with Preferences for the Timing of Resolution of Uncertainty," Economic Theory, 9, 3-22. 
Epstein, L., and S. Zin, 1989, "Substitution, Risk Aversion, and the Temporal Behavior of Consumption Growth and Asset Returns I: A Theoretical Framework," Econometrica, 57, 937-969.

Gennotte, G., 1986, "Optimal portfolio choice under incomplete information," Journal of Finance, $41,733-746$.

Hamilton, J. D., 1994, Time Series Analysis, Princeton University Press, New Jersey.

Hansen, L. P., J. C. Heaton, and N. Li, 2005, "Consumption Strikes Back? Measuring Long Run Risk," Working Paper, University of Chicago.

Karatzas, I., and S. E. Shreve, 1991, Brownian Motion and Stochastic Calculus, Springer-Verlag, New York, 2nd edn.

Kreps, D. M., and E. L. Porteus, 1978, "Temporal Resolution of Uncertainty and Dynamic Choice Theory," Econometrica, 46, 185-200.

Liptser, R. S., and A. N. Shiryayev, 2001, Statistics of Random Processes, Springer-Verlag, New York, New York.

Lucas, Jr., R. E., 1978, "Asset Prices in an Exchange Economy," Econometrica, 46, 1426-14.

Piazzesi, M., and M. Schneider, 2006, "Equilibrium Yield Curves," NBER Macroeconomics Annual.

Skiadas, C., 1998, "Recursive utility and preferences for information," Economic Theory, 12, 293312.

Tauchen, G., and R. Hussey, 1991, "Quadrature Based Methods for Obtaining Approximate Solutions to Nonlinear Asset Pricing Models.," Econometrica, 59, 371-396.

Veronesi, P., 1999, "Stock Market Overreaction to Bad News in Good Times: A Rational Expectations Equilibrium Model," Review of Financial Studies, 12, 975-1007.

Veronesi, P., 2000, "How Does Information Quality Affect Stock Returns?," Journal of Finance, $55,807-837$.

Wang, J., 1993, "A Model of Intertemporal Asset Prices Under Asymmetric Information," Review of Economic Studies, 60, 249-282.

Weil, P., 1990, "Non-Expected Utility in Macroeconomics," Quarterly Journal of Economics, 105, $29-42$.

Wonham, W. M., 1964, "Some Applications of Stochastic Differential Equations to Optimal Nonlinear Filtering," SIAM Journal on Control, 2, 347-369. 